\title{
Indoor Positioning Using GPS Revisited
}

Mikkel Baun Kjargaard, Henrik Blunck, Torben Godsk, Thomas Toftkjaer , Dan Lund Christensen, and Kaj Grønbak
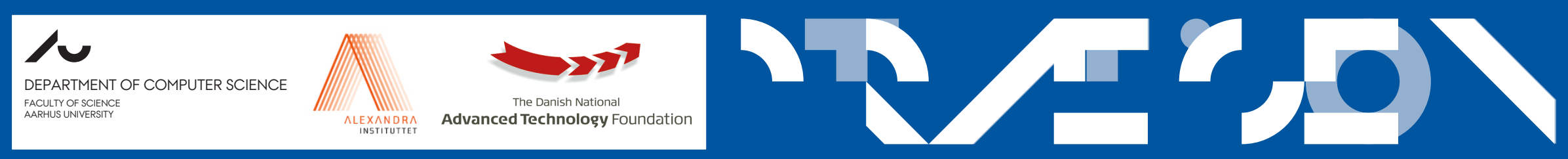


\section{Overview}

\section{> Motivation}

> GPS and alternative positioning technologies

> Recent advances: High-Sensitivity and Assisted GPS

> Evaluating GPS performance indoors

> Measurement campaign results

> The future in GPS and indoor positioning 


\section{MOTIVATION}

> Applications require positioning with indoor coverage

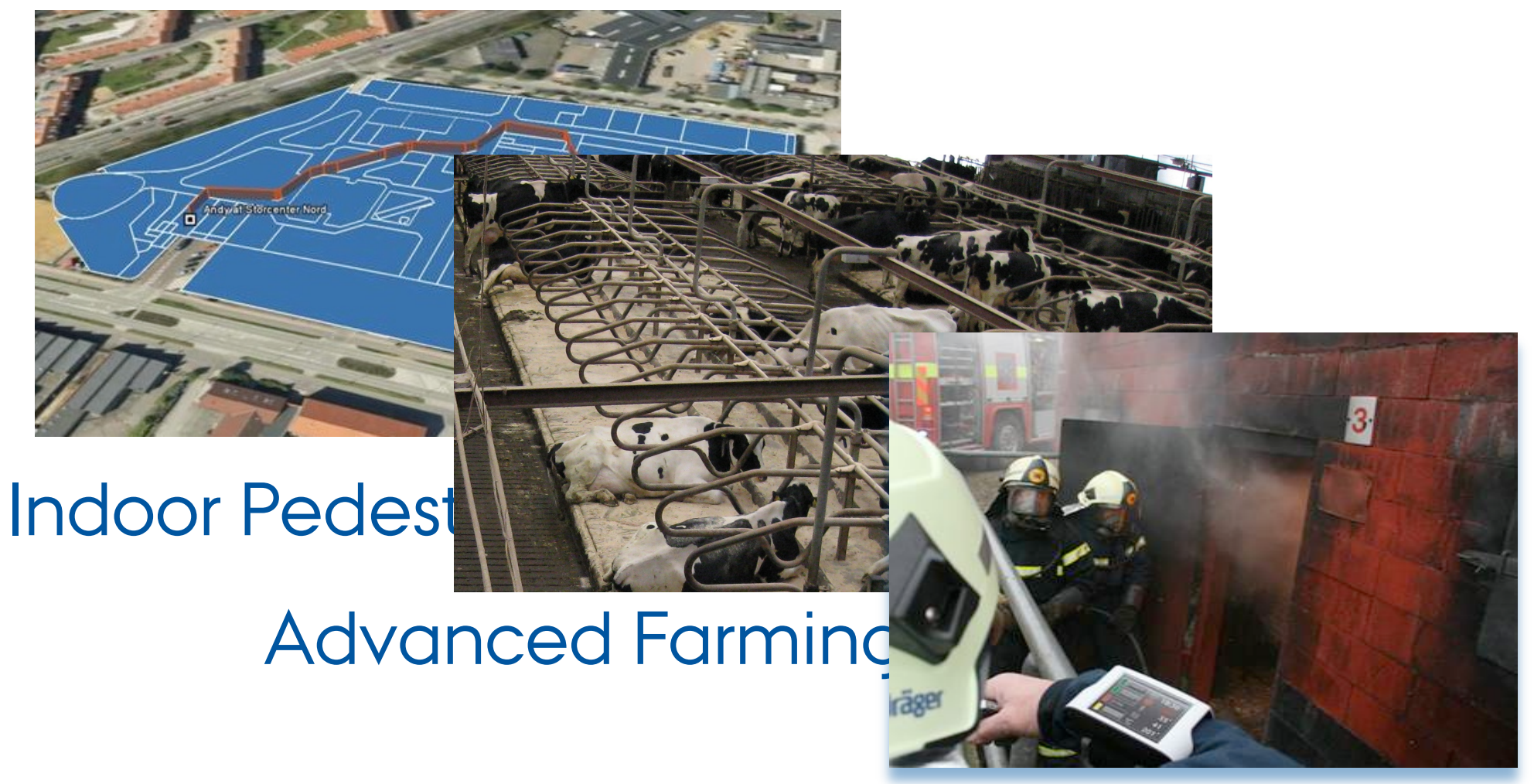

Search\&Rescue: Coordinating Fire Fighters

> Scenarios from a pervasive positioning project run at

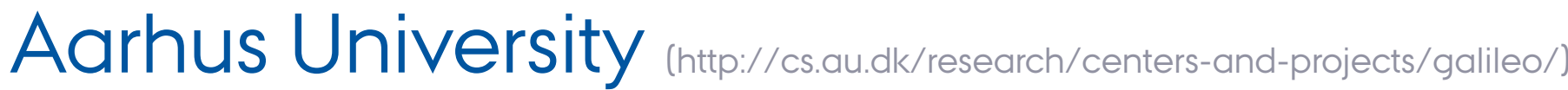




\section{MOTIVATION}

1. Pervasive Coverage

> Applications work where needed

2. Independence from Local Infrastructures

> No need to setup and maintain local infrastructure.

> Reliability (e.g. fire fighting)

> Global Navigation Satellite Systems (e.g. GPS)

> Satisfying 2. (Space segment, Control segment, A-GPS)

> Previously GPS did only work outdoors and therefore did not satisfy 1 .

> How are the advances in technology changing this situation? 


\section{Overview}

> Motivation

> GPS and alternative positioning technologies

> Recent advances: High-Sensitivity and Assisted GPS

> Evaluating GPS performance indoors

> Measurement campaign results

> The future in GPS and indoor positioning 


\section{GPS and Alternative Technologies}
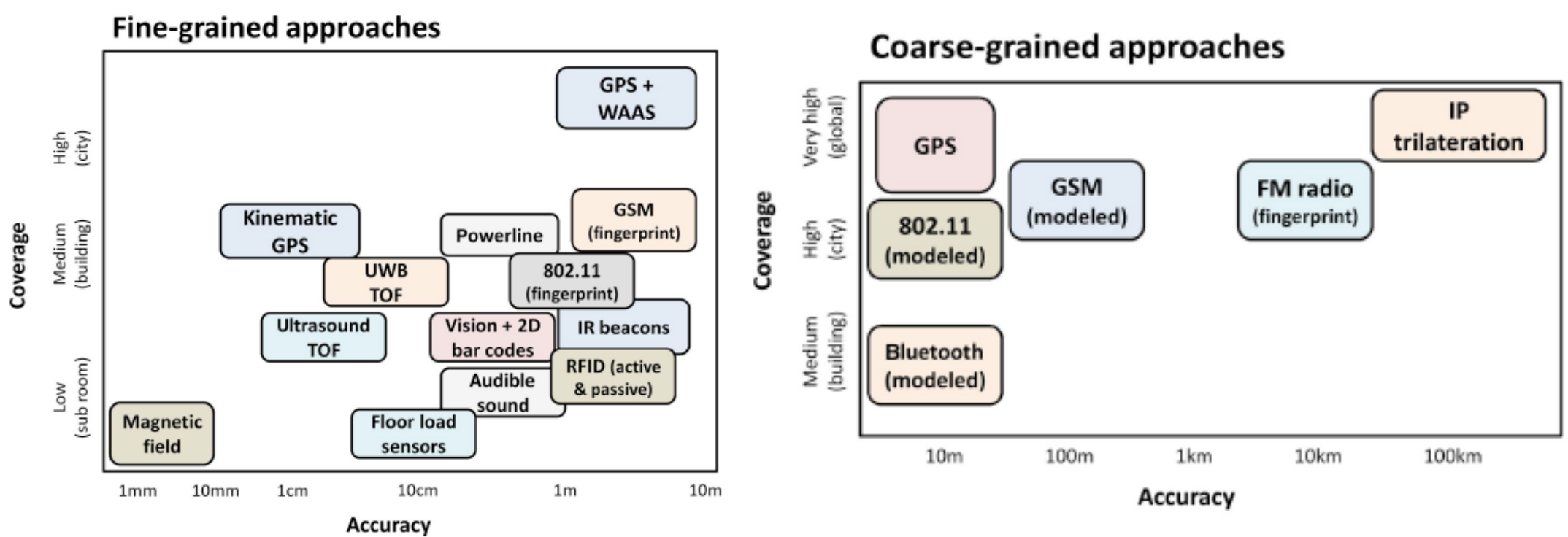

Anthony LaMarca, Eyal de Lara.d Location Systems: An Introduction to the Technology Behind Location Awareness, Morgan \& Claypool Publishers, 2008

\section{> Inertial Positioning}

Oliver Woodman, Robert Harle: RF-Based Initialisation for Inertial Pedestrian Tracking. Pervasive 2009: 238-255 


\section{Overview}

> Motivation

> GPS and alternative positioning technologies > Recent advances: High-Sensitivity and Assisted GPS

> Evaluating GPS performance indoors

> Measurement campaign results

> The future in GPS and indoor positioning 


\section{High-Sensitivity and Assisted GPS}

>Problem - Tune into the signals

$>$ A search problem where we have to find a peak

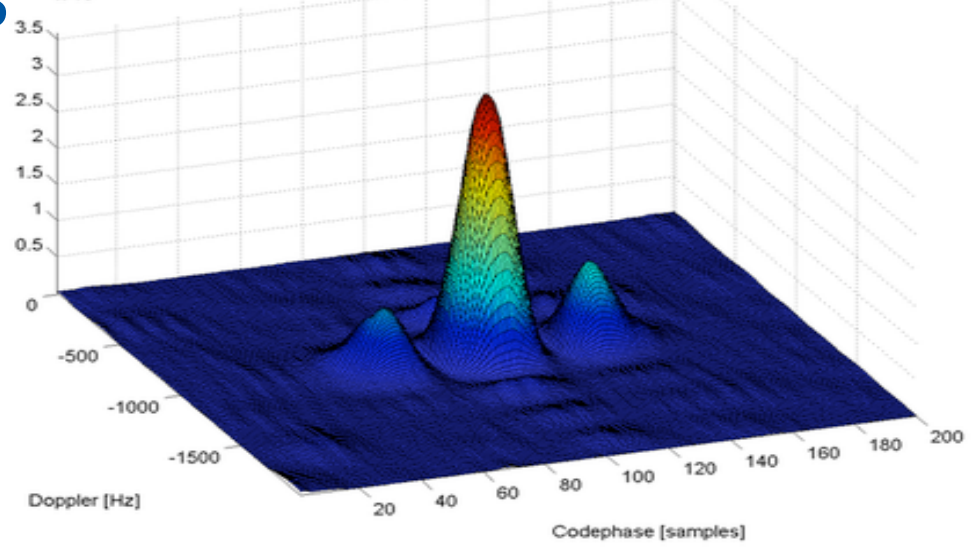

> In weak signal conditions the peak is very small

-> disappears in background noise

$>$ High-Sensitivity

> Integrate signals over time to make the peak higher

> Made possible by massive parallelization

> Old outdoor receiver: $\mathbf{3 6}$ correlations per second

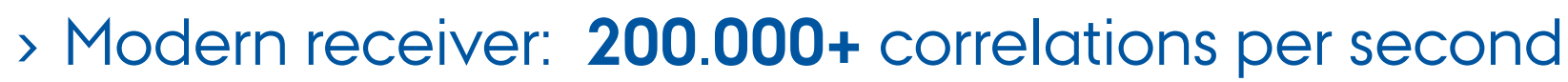




\section{High-Sensitivity and Assisted GPS}

> Assisted GPS (A-GPS)

> Providing the receiver with assistence data over other communication means (e.g. cellular networks)

$>$ Help with reducing the search space by

> Providing rough current time (+/- 2 seconds)

$>$ Providing rough position ( $<3$ kilometers)

> Problem - Decoding navigation data

> Navigation data needed to compute receiver position

> In weak signal conditions the receiver cannot decode these data bits from the satellite signals.

$>$ A-GPS

> Navigation data provided as part of the assistance data 


\section{Overview}

> Motivation

> GPS and alternative positioning technologies

> Recent advances: High-Sensitivity and Assisted GPS

> Evaluating GPS performance indoors

> Measurement campaign results

> The future in GPS and indoor positioning 


\section{GPS receivers employed in campaign}

$>2$ classes of receivers were investigated:

$>$ dedicated GPS receivers

$>$ in-phone receiver systems

$>\rightarrow$ size, cost and power restrictions

> Setup enforces limits on \# receivers

$>$ to avoid near-field interactions between receivers

$>\rightarrow$ Example devices chosen for each class:

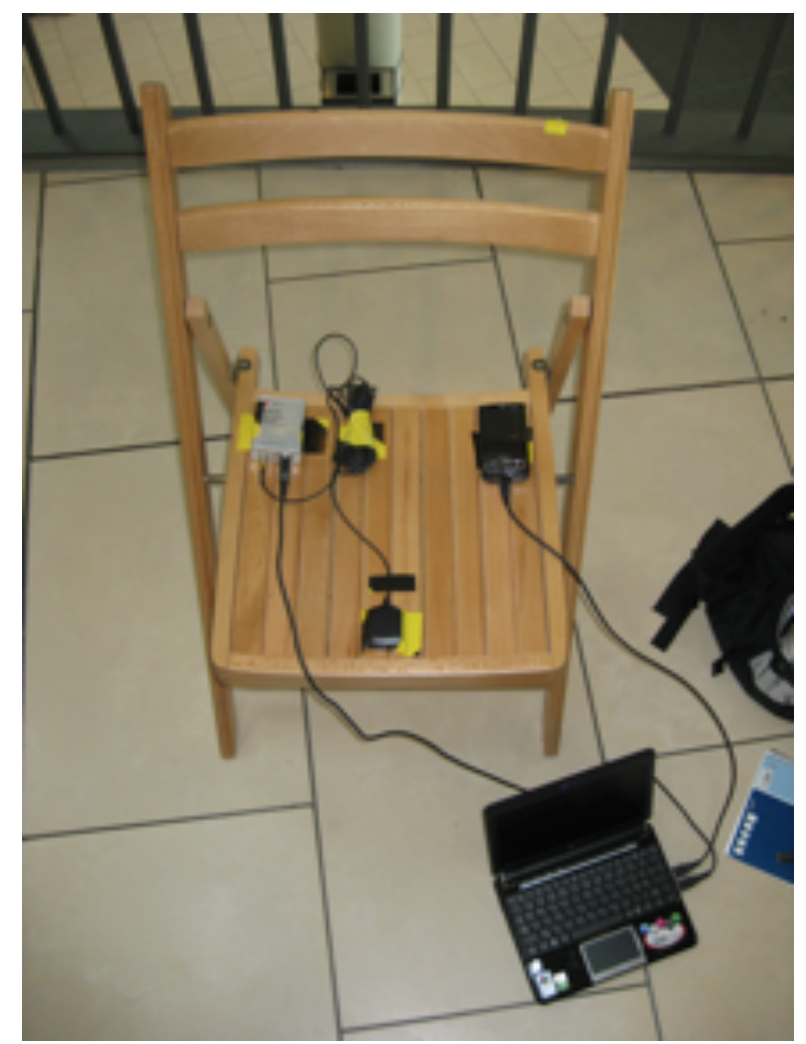

> dedicated U-blox LEA + external active patch antenna

> Nokia N95 in-phone GPS system

> Texas Instruments GPS chip + internal active patch antenna 


\section{Indoor environments investigated}

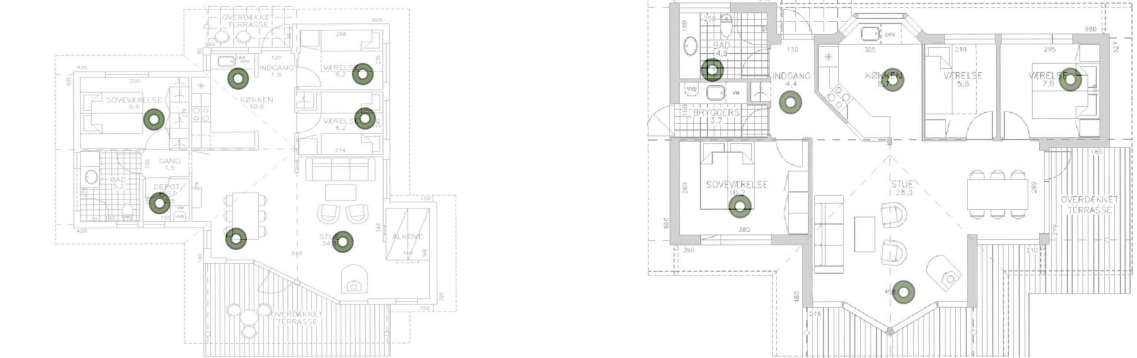

wooden houses
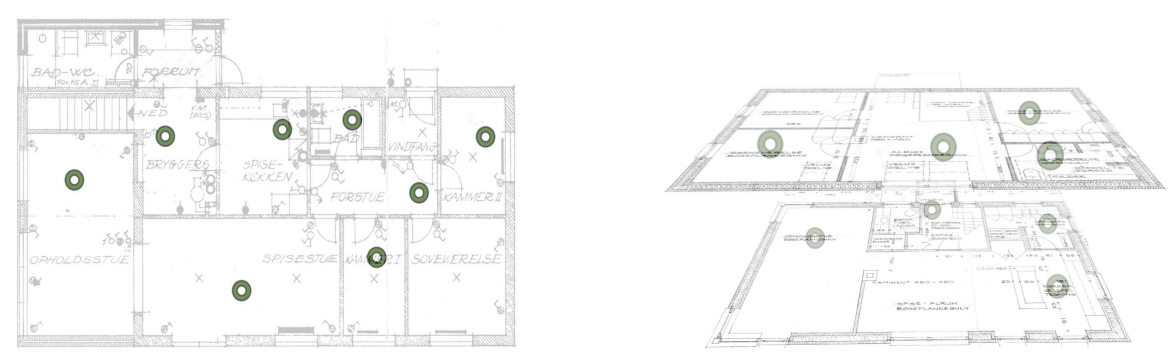

brick houses

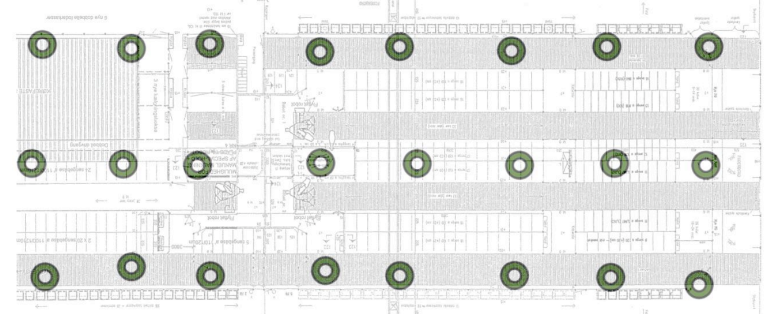

Warehouse

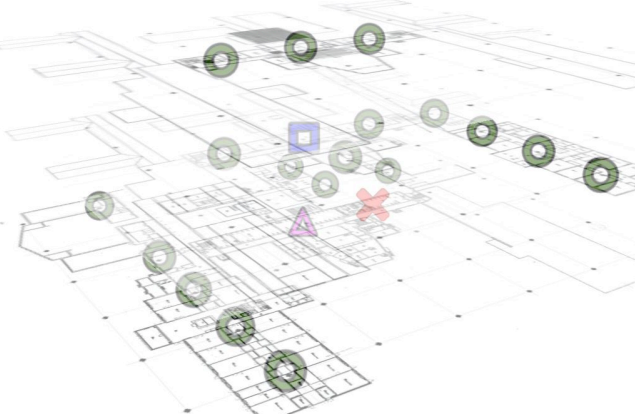

School building

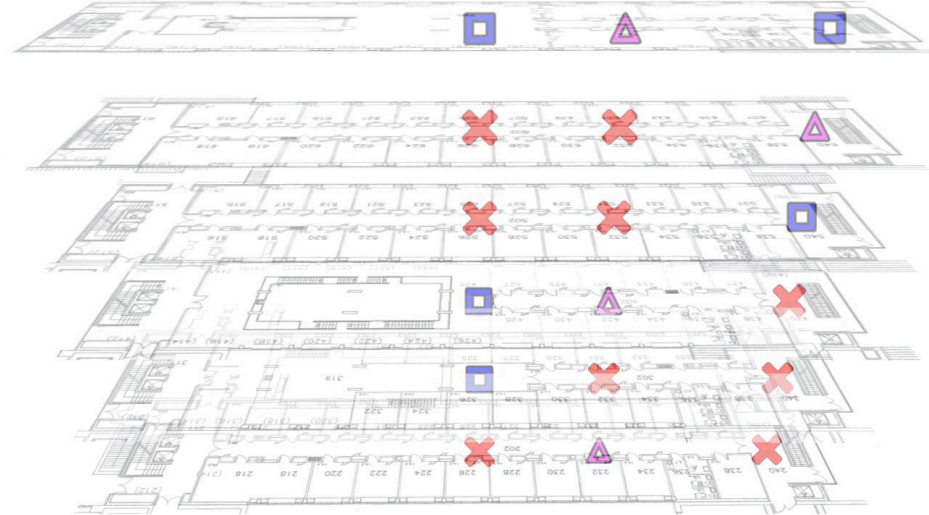

Tower block 


\section{Overview}

> Motivation

> GPS and alternative positioning technologies

> Recent advances: High-Sensitivity and Assisted GPS

> Evaluating GPS performance indoors

> Measurement campaign results

> The future in GPS and indoor positioning 


\section{Indoor GPS performance levels measured}

>.. using a dedicated receiver, e.g. u-blox's

\section{$>$ Availability and time-to-first-fix}

> non-assisted GPS:

> often not available deep indoors; often TTFF > 1 min

$>$ A-GPS:

> better availability and often TTFF < $5 \mathrm{sec}$ (deep indoors: up to $3 \mathrm{~min}$ )

> Positioning accuracy (in 2D RMS; using A-GPS)

$>$ in smaller wooden / brick houses: 4m / 7m

$>$ up to 60m "deep indoors" in tower block

$>$ Low accuracies caused by:

> signal attenuation $\rightarrow$ (poor constellations of) only few "visible" satellites

$>$ multipath phenomena (i.e. strong signal echoes) 


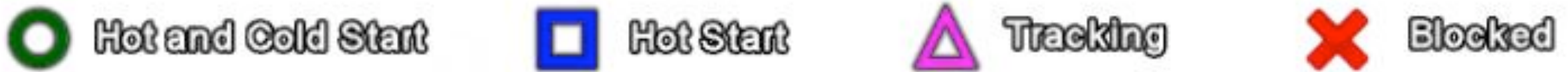

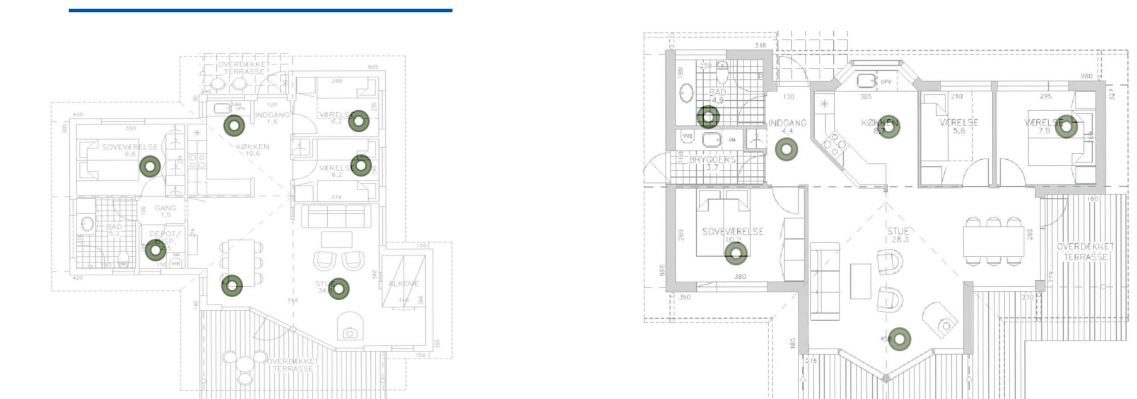

wooden houses
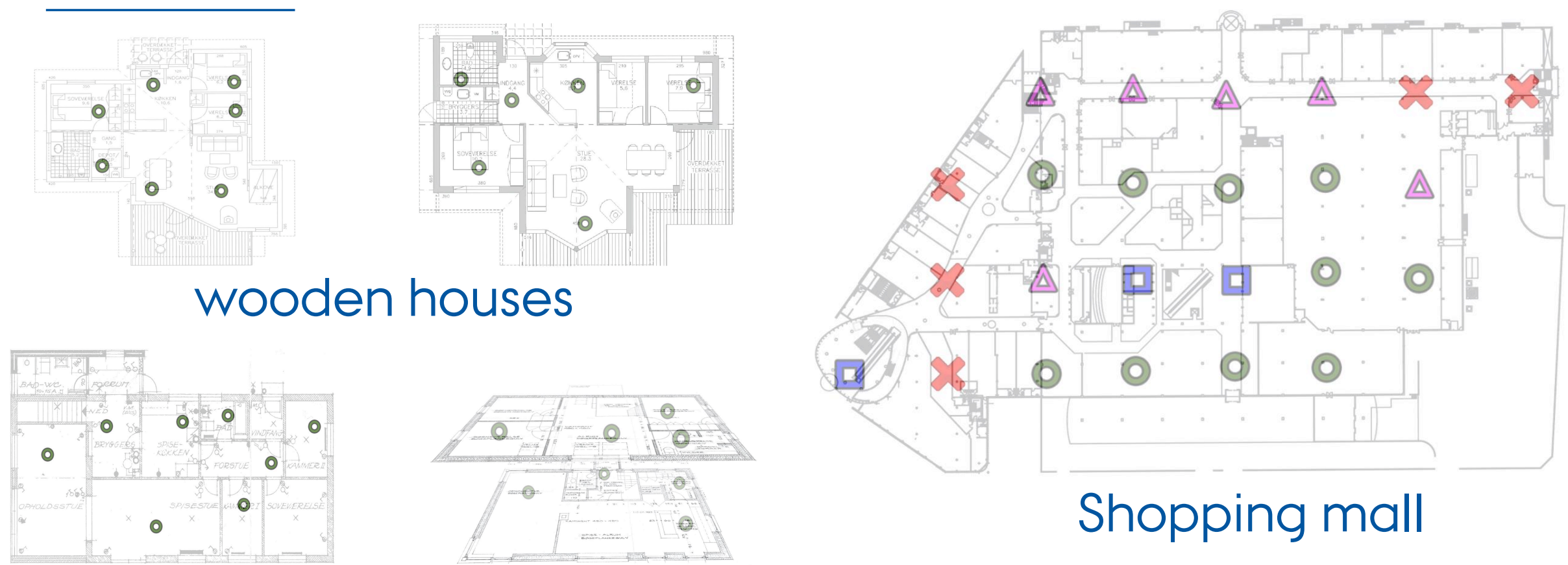

brick houses

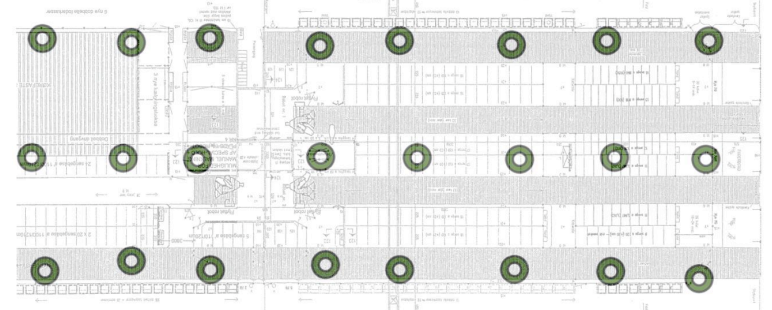

Warehouse

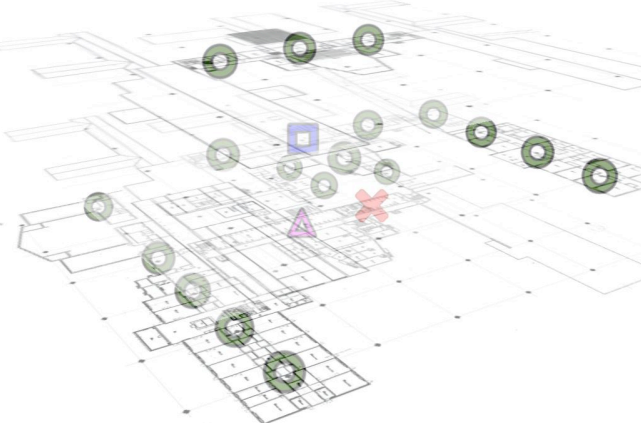

School building

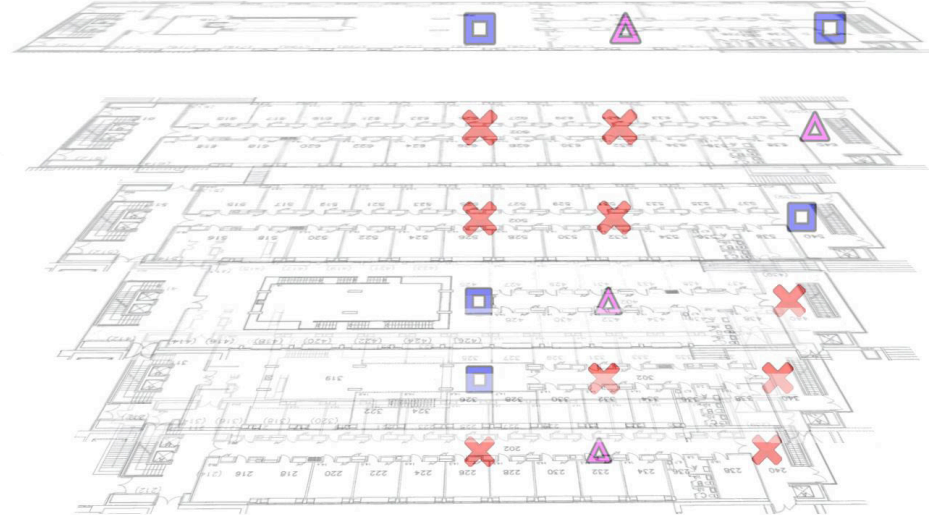

Tower block 


\section{- Hroband ard Stan \\ - Morstar \\ $\Delta$ Ttracklar \\ is Blocked}

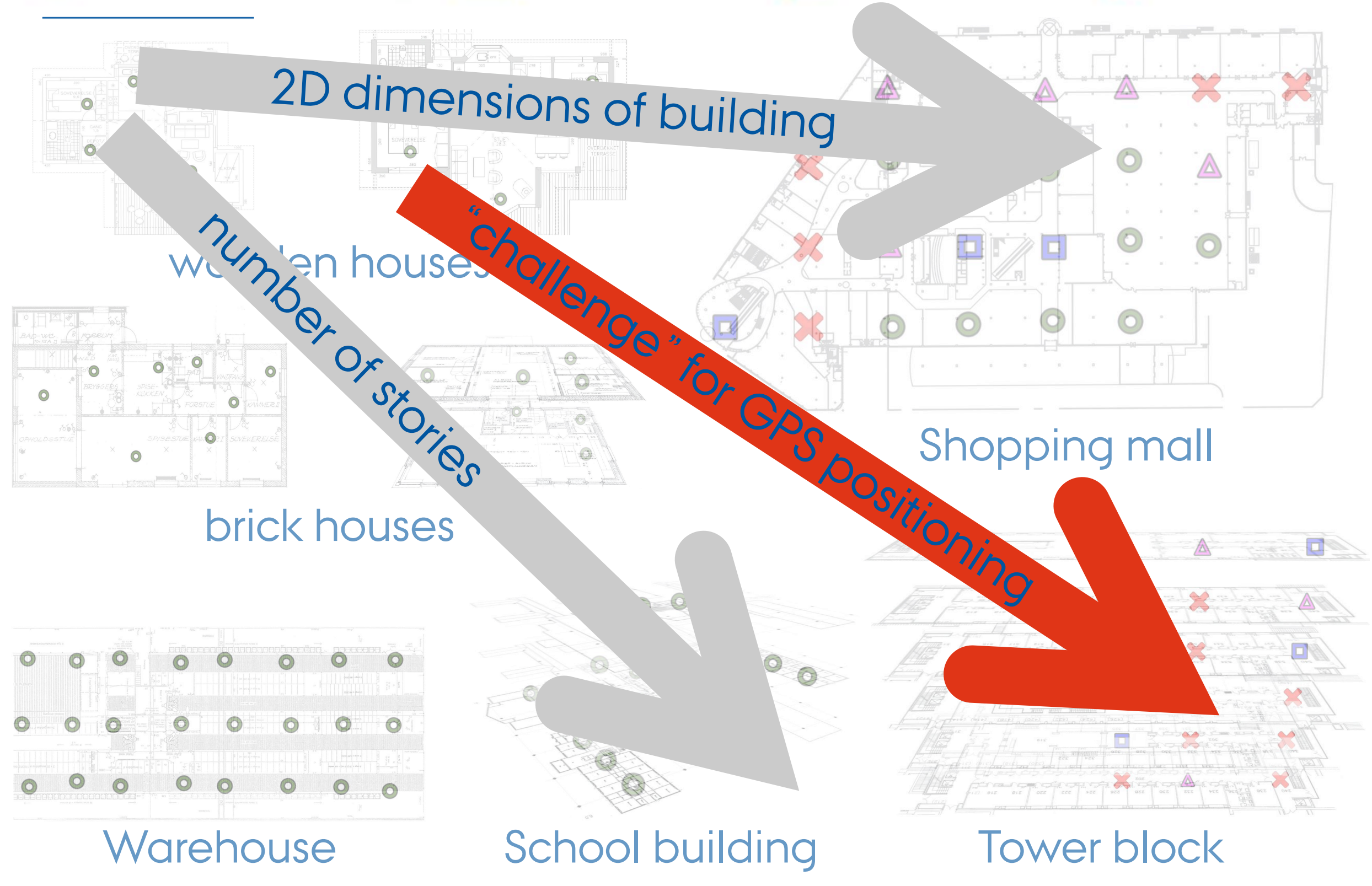




\section{GPS signal-to-noise-ratios}

>Problem - Tune into the signals

> A search problem where we have to find a peak

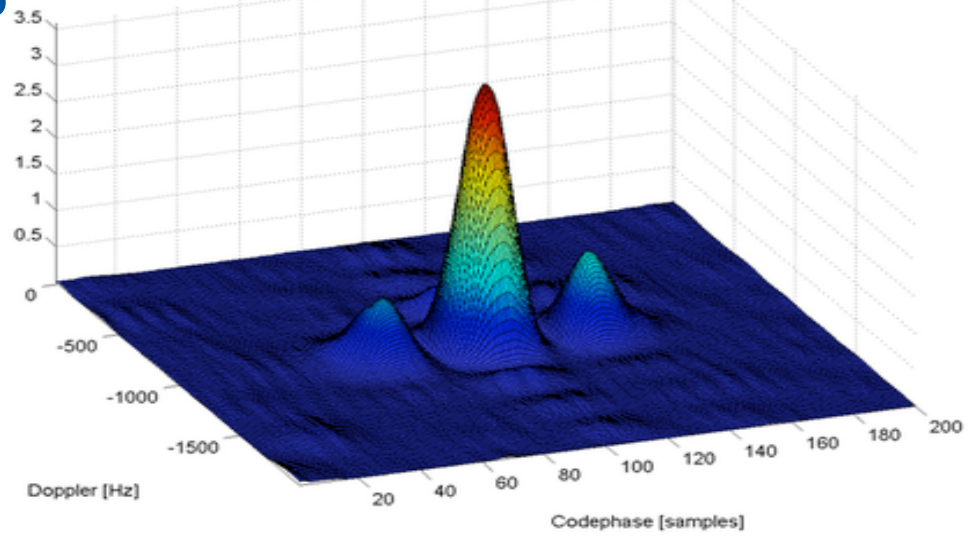

> In weak signal conditions the peak is very small

-> disappears in background noise

> GPS Satellite signal is sent with only 27W

> Noise levels at receiver: -200 dBW/Hz

$>$ GPS signal strength at receiver:

> outdoors: - $155 \mathrm{dBW} \rightarrow$ SNR: $45 \mathrm{~dB} / \mathrm{Hz}$

> deep indoors: often down to $185 \mathrm{dBW} \rightarrow \mathrm{SNR}: 15 \mathrm{~dB} / \mathrm{Hz}$ i.e. 1000 times weaker than outdoors 


\section{Indoor GPS Performance: Availability}

\section{> Main factor impairing availability:}

> obstructing building elements

> cause signal attenuation

\begin{tabular}{|c|c|c|c|c|c|c|}
\hline \multirow[b]{2}{*}{ Building Type } & \multicolumn{4}{|c|}{ Walls } & \multirow[b]{2}{*}{ Roof } & \multirow[b]{2}{*}{ dB } \\
\hline & External & $\mathbf{d B}$ & Internal & dB & & \\
\hline Wood 1 & Wood & 2.40 & Wood & 2.40 & Tiles & 5.19 \\
\hline Wood 2 & Wood & 2.40 & Wood & 2.40 & Tiles & 5.19 \\
\hline Brick 1 & Double Brick & 10.38 & Brick & 5.19 & Fiber Cement & N/A \\
\hline Brick 2 & Double Brick & 10.38 & Brick & 5.19 & Tiles & 5.19 \\
\hline $\begin{array}{l}\text { School } \\
\text { main building + right wing } \\
\text { annex }+ \text { left wing }\end{array}$ & $\begin{array}{l}\text { Double Brick } \\
\text { Brick and Concrete }\end{array}$ & $\begin{array}{l}10.38 \\
14.76\end{array}$ & \begin{tabular}{|l} 
Brick \\
Concrete
\end{tabular} & $\begin{array}{l}5.19 \\
9.57\end{array}$ & $\begin{array}{l}\text { Tiles } \\
\text { Tiles }\end{array}$ & $\begin{array}{l}5.19 \\
5.19\end{array}$ \\
\hline Warehouse & $\begin{array}{l}\text { Fiber Cement and } \\
\text { Curtains/Openings }\end{array}$ & $\begin{array}{l}\text { N/A } \\
\text { N/A }\end{array}$ & Equipment & N/A & Fiber Cement & N/A \\
\hline Shopping Mall & $\begin{array}{l}\text { Reinforced Concrete } \\
\text { Tinted Glass } \\
\text { Glass }\end{array}$ & \begin{tabular}{r|}
16.70 \\
24.44 \\
2.43
\end{tabular} & Brick & 5.19 & \begin{tabular}{|l} 
Flagstone \\
Sand \\
Felt roofing \\
Concrete
\end{tabular} & $\begin{array}{r}\text { N/A } \\
2 \\
\text { N/A } \\
9.57\end{array}$ \\
\hline Tower block & $\begin{array}{l}\text { Double Brick } \\
\text { around Concrete }\end{array}$ & 19.95 & Brick & 5.19 & Tiles & 5.19 \\
\hline
\end{tabular}




\section{Indoor GPS Performance: Availability}

> Main factor impairing availability:

> obstructing building elements

> cause signal attenuation (additive)

$>$.. and dispersion $\rightarrow$ user's distance from elements crucial

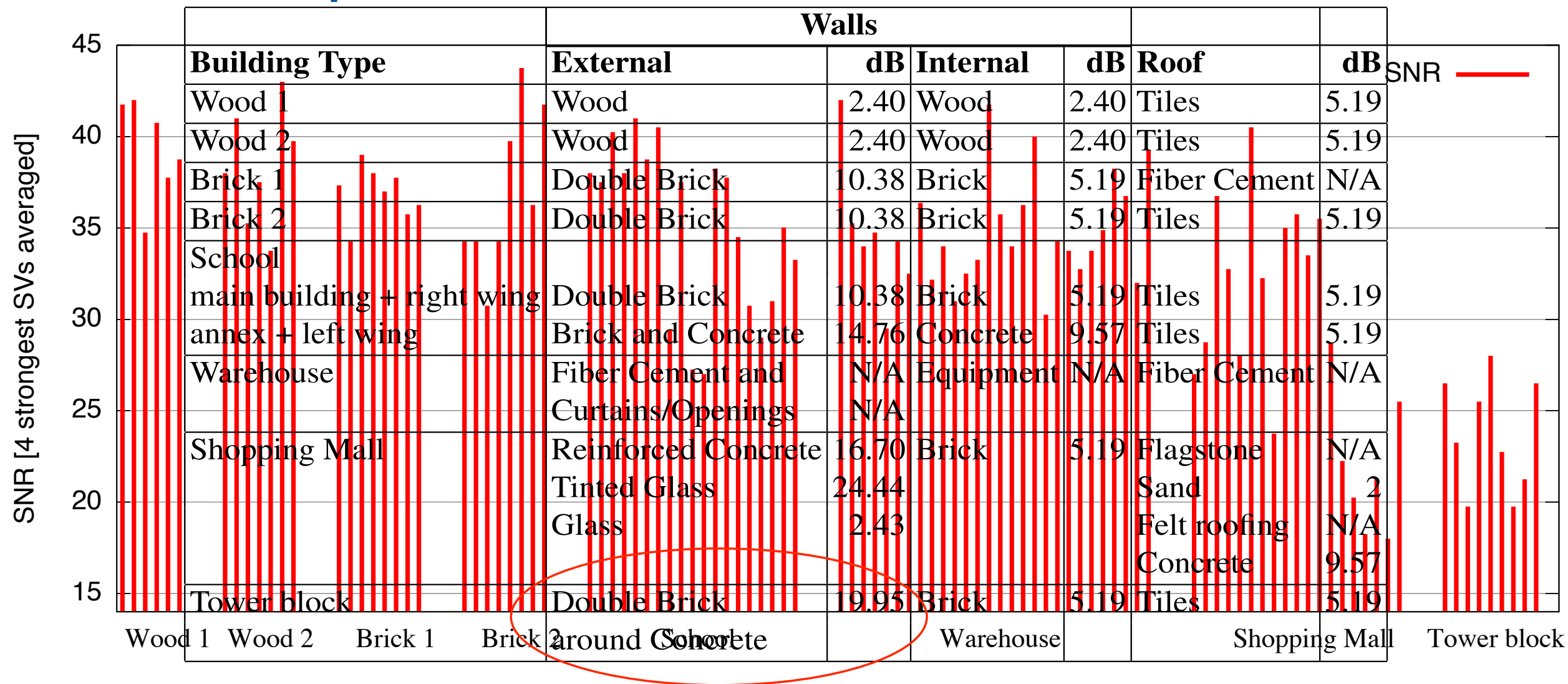




\section{Challenges in Indoor GPS Positioning}

> Outdoors: Satellites overhead received stronger

> Less so indoors; esp. on lower floors of tall buildings

> signal (versions) stronger through windows than through roof

> echoes cause pseudorange errors!

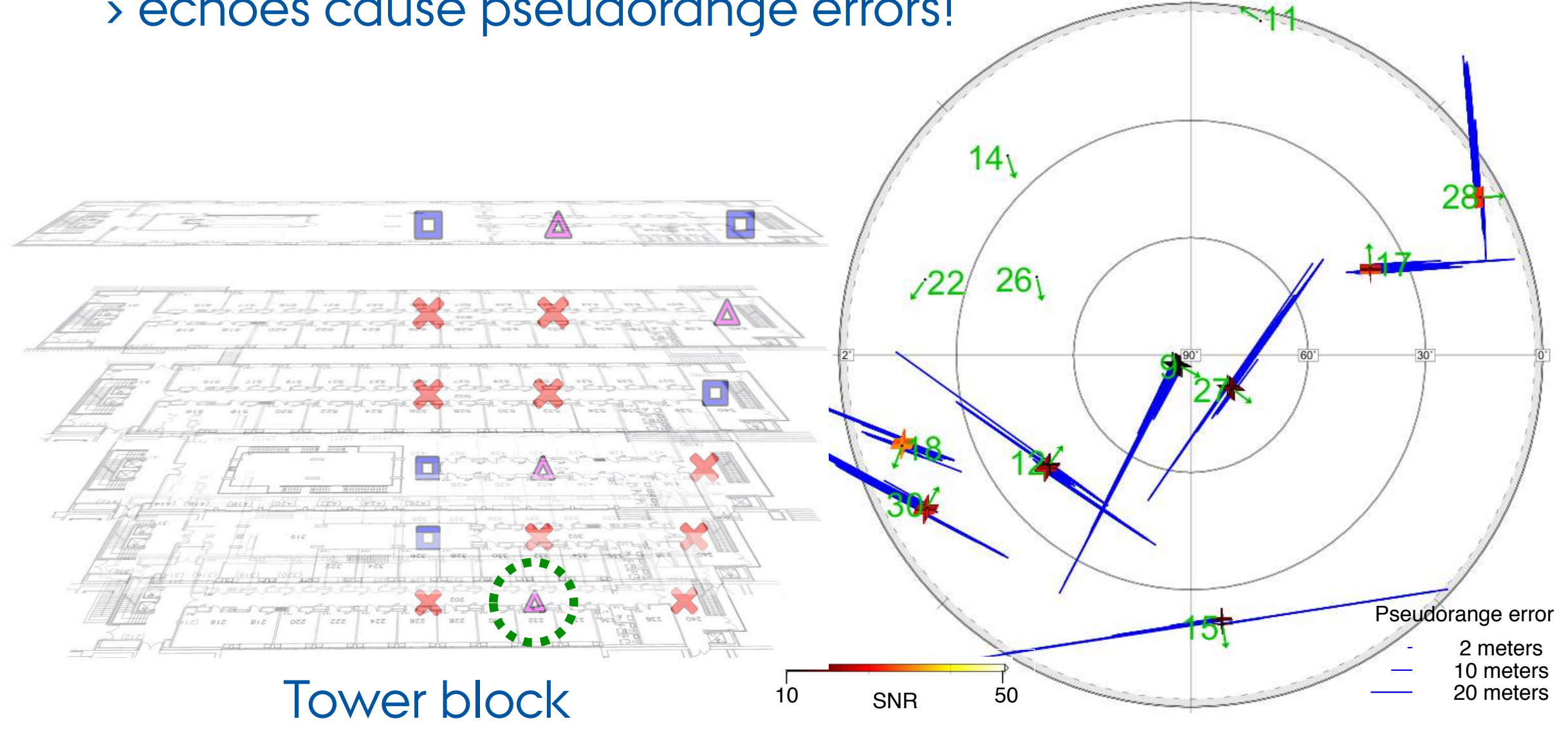




\section{Challenges in Indoor GPS Positioning}

> Outdoors: Satellites overhead received stronger

> Less so indoors; esp. on lower floors of tall buildings

> signal (versions) stronger through windows than through roof

> echoes cause pseudorange errors!

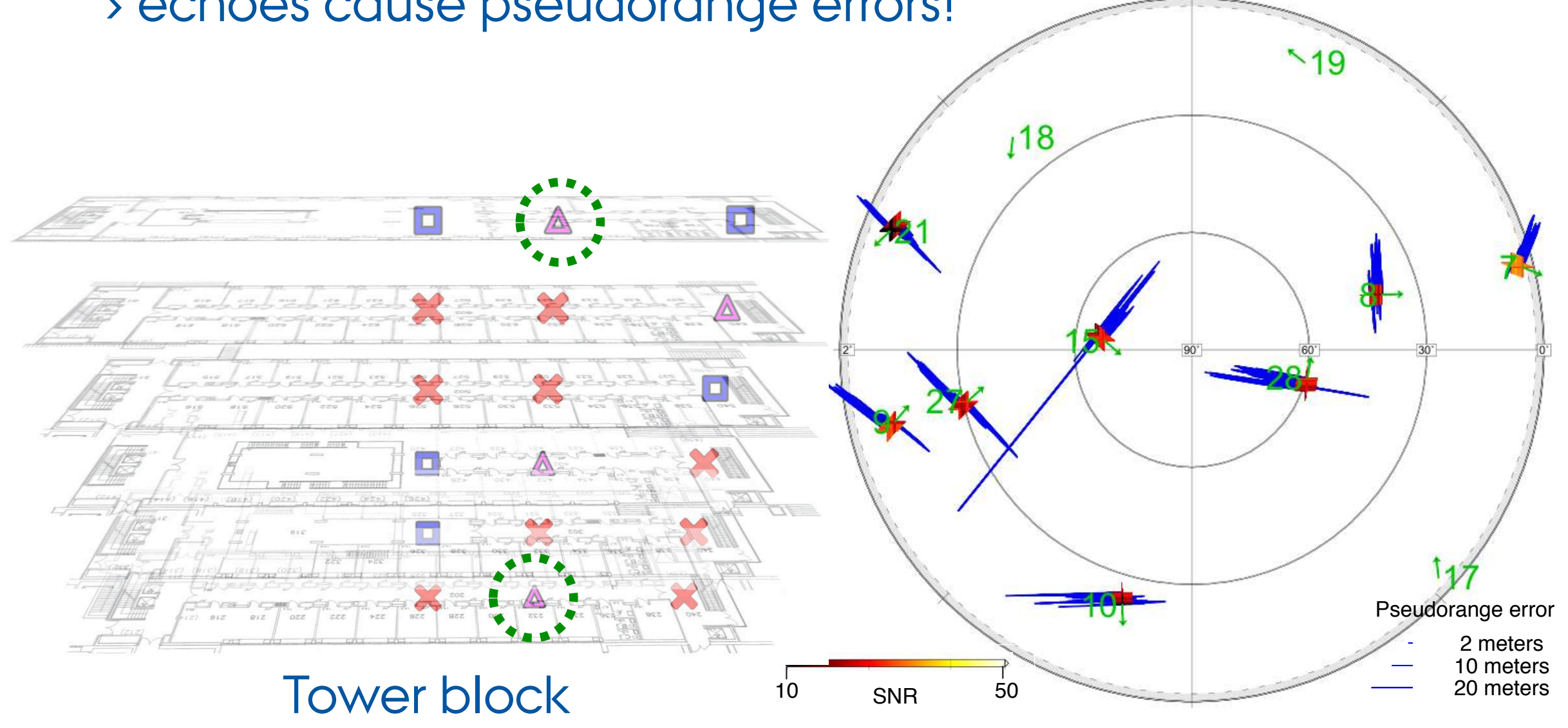




\section{- crosend oord staris \\ arossteris \\ $\Delta$ Ttrakstho \\ X Blooked}

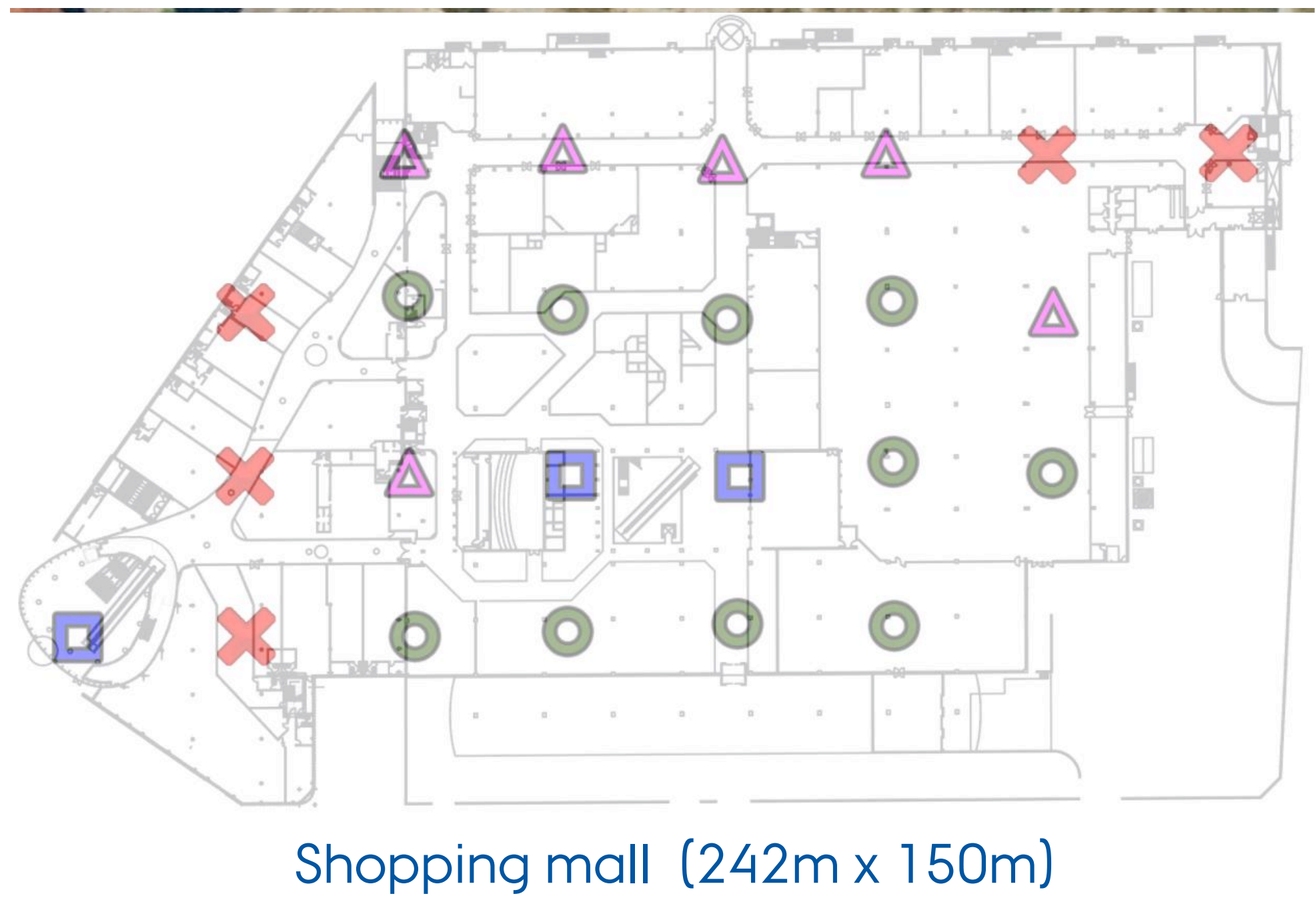

"signals are wanted strong... but if they are: don't trust them" 


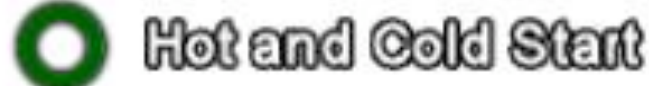 \\ - arosisters \\ $\Delta$ Treakstag \\ X Blooked}

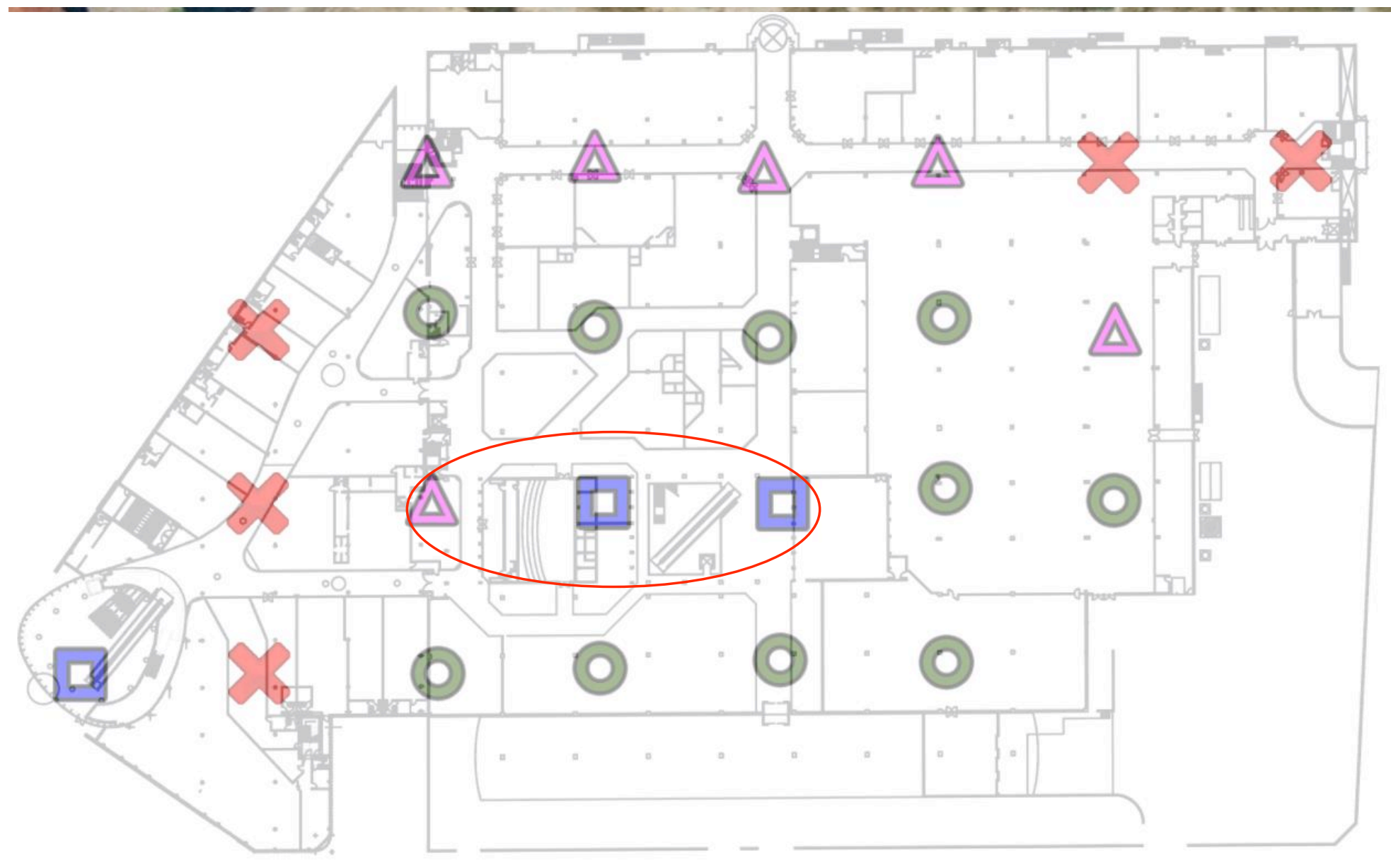

Shopping mall $(242 \mathrm{~m} \times 150 \mathrm{~m})$ 


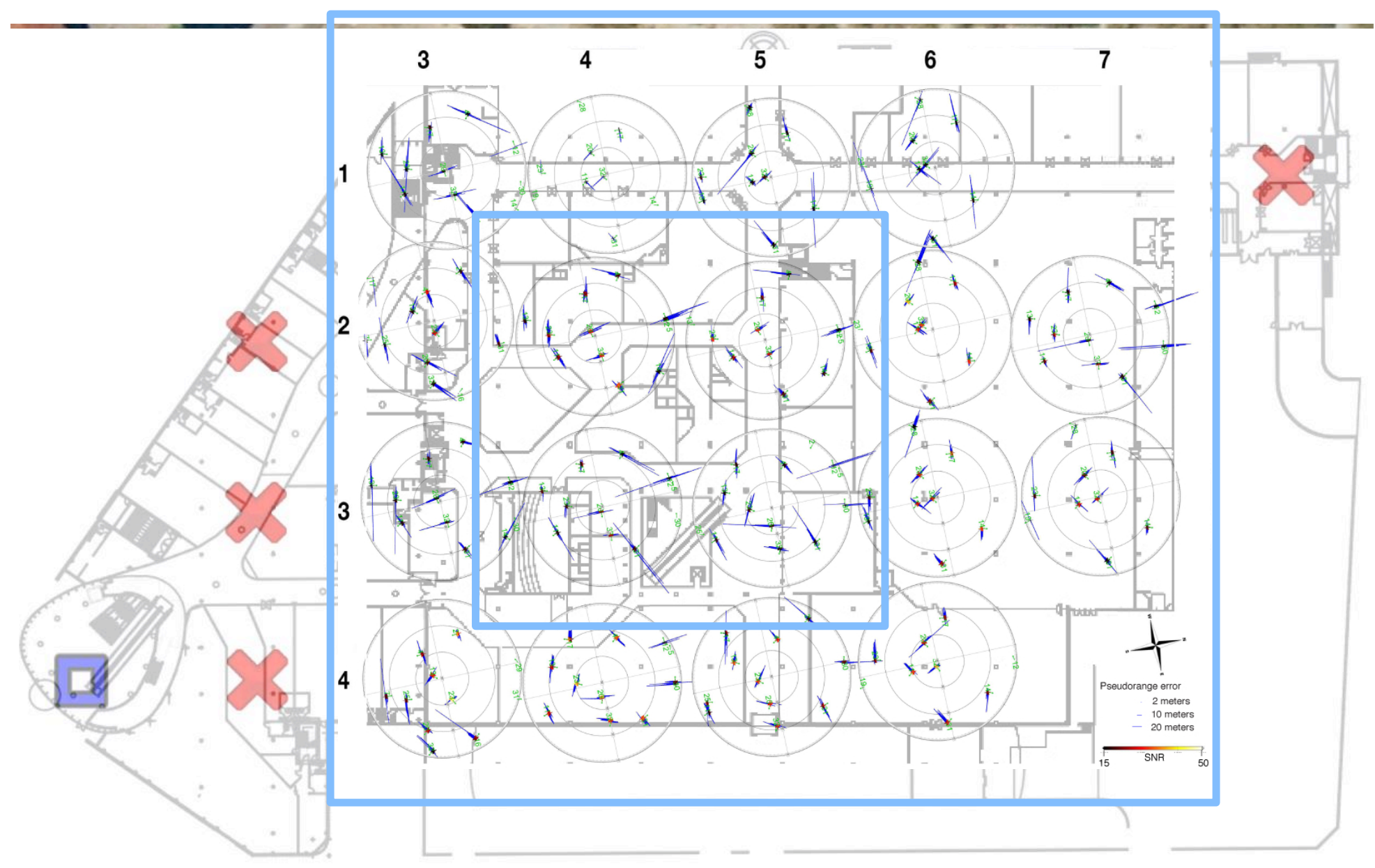

Shopping mall (242m x 150m) 


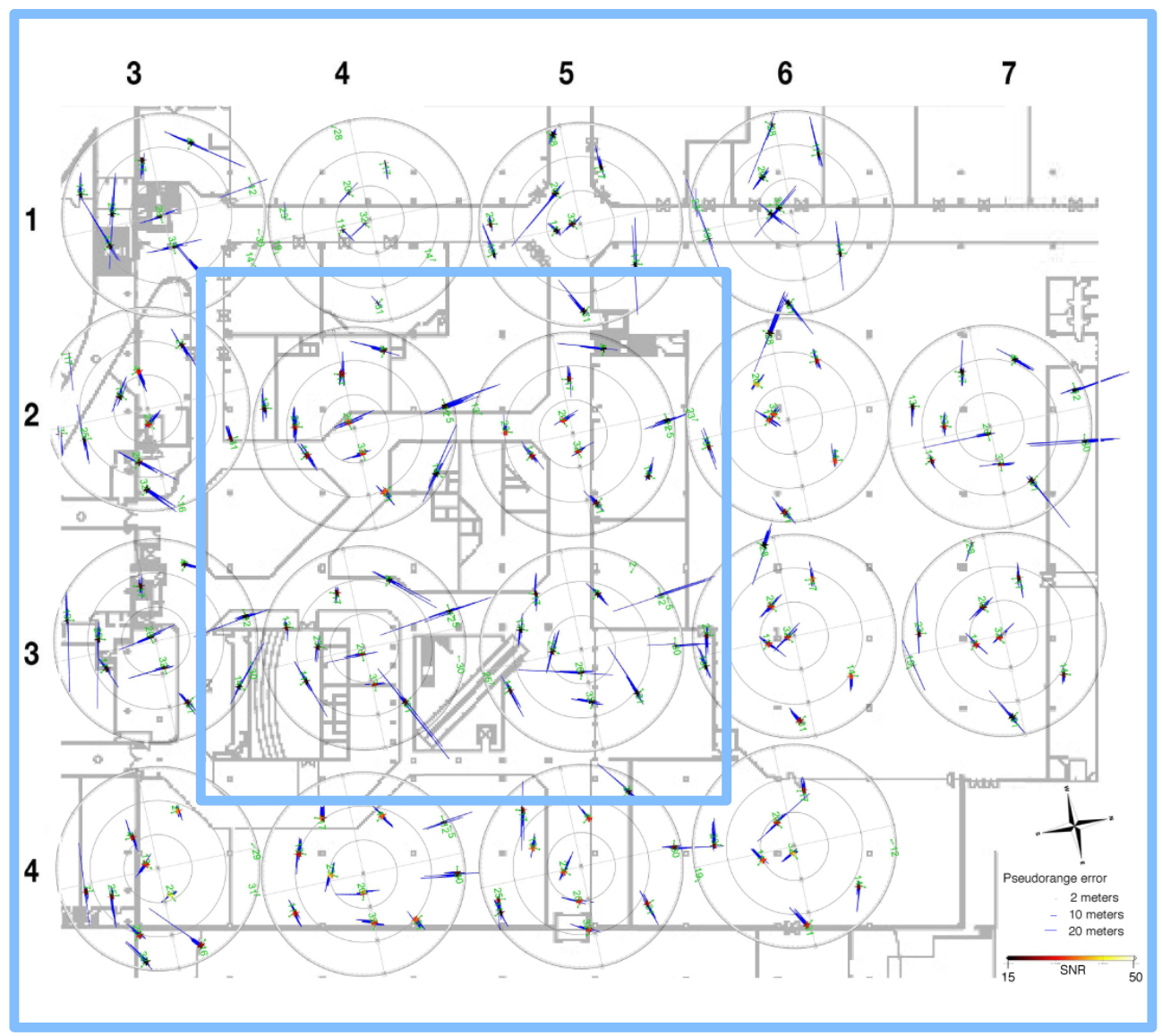




\section{GPS performance: Availability/Accuracy}

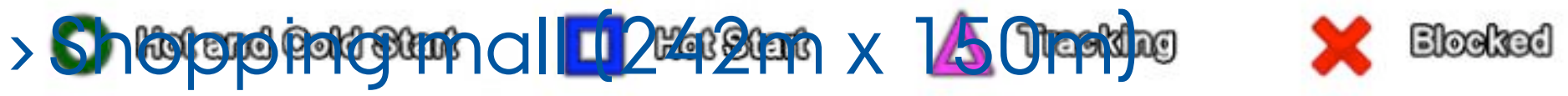

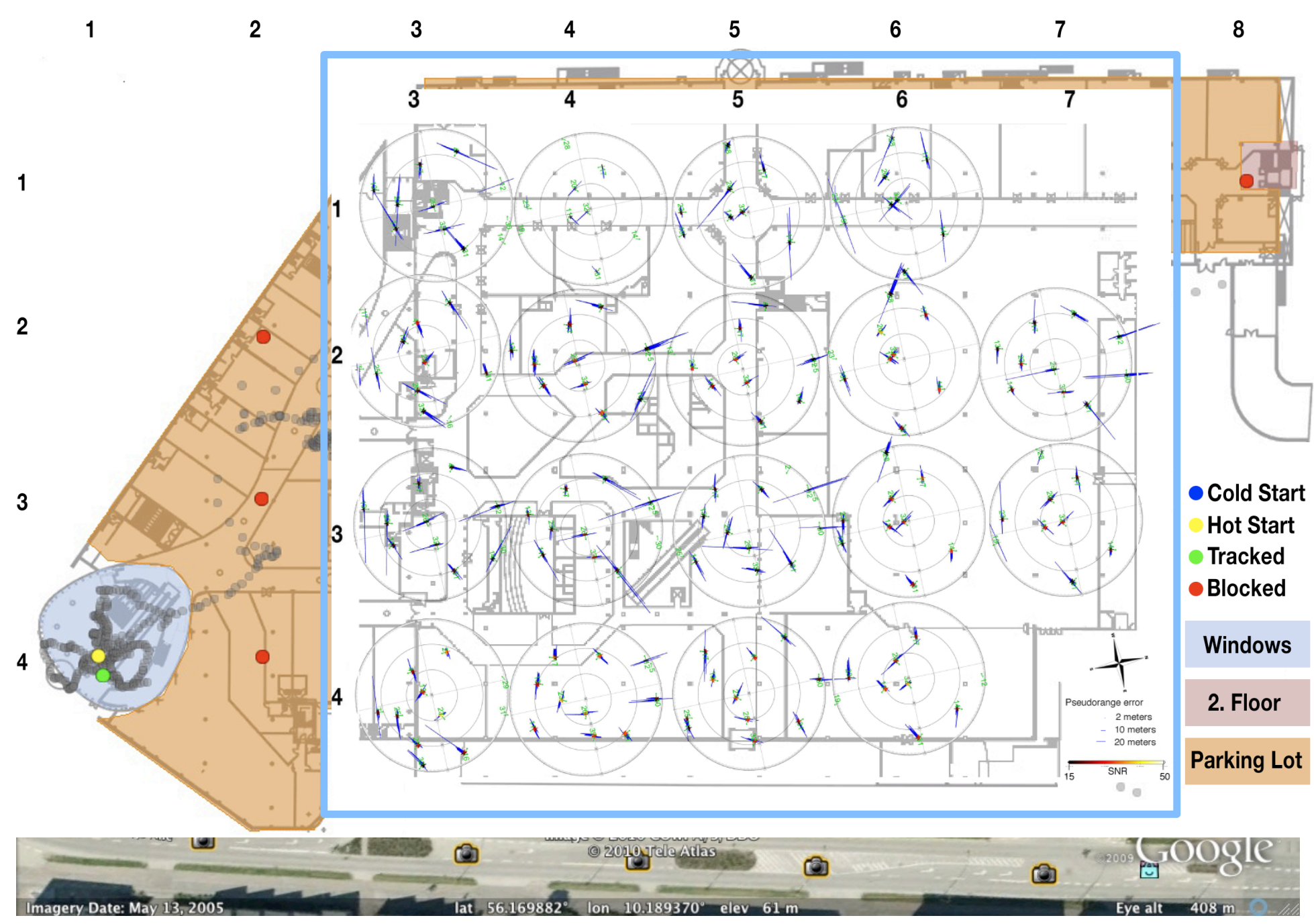




\section{Indoor GPS Performance: Accuracy (in RMS)}

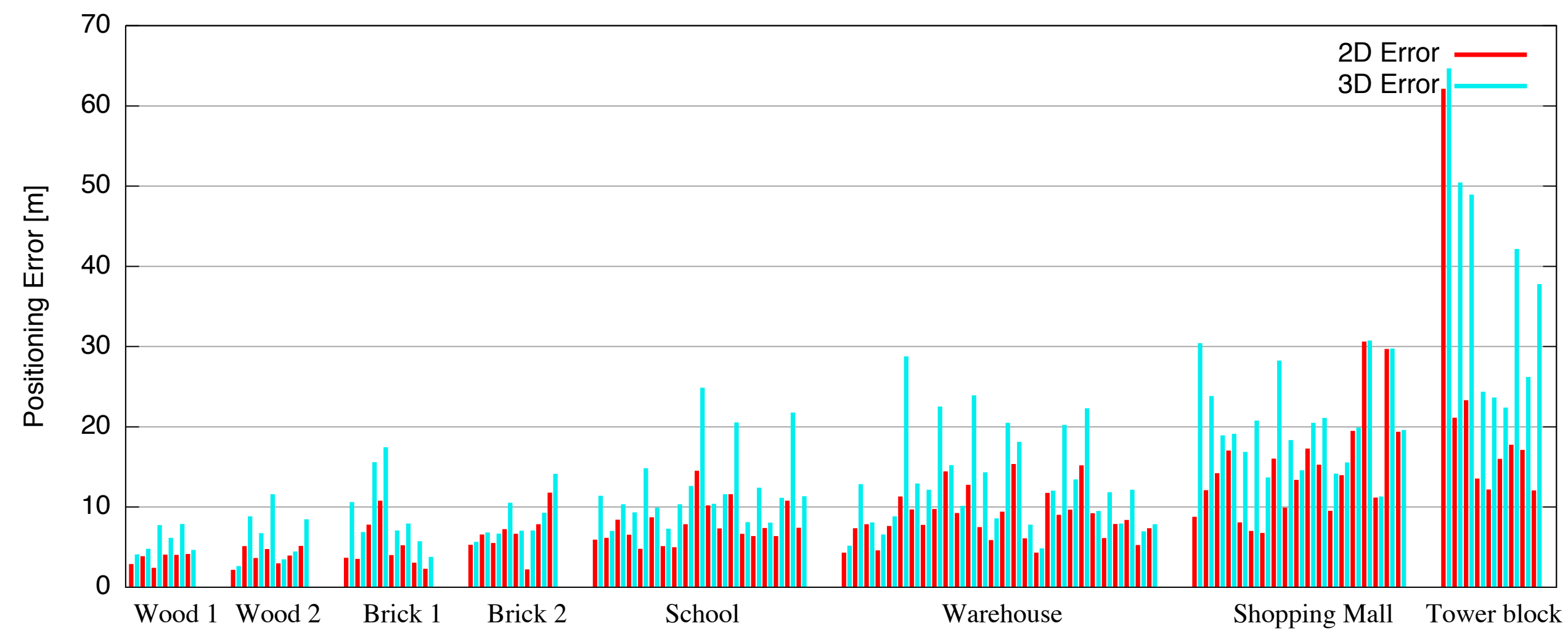

> Outdoors: $3 \mathrm{~m}$

> In houses on average: $\sim 5 \mathrm{~m}$

> In shopping mall on average: $\sim 15 \mathrm{~m}$ 


\section{Indoor GPS Performance: Accuracy (in RMS)}

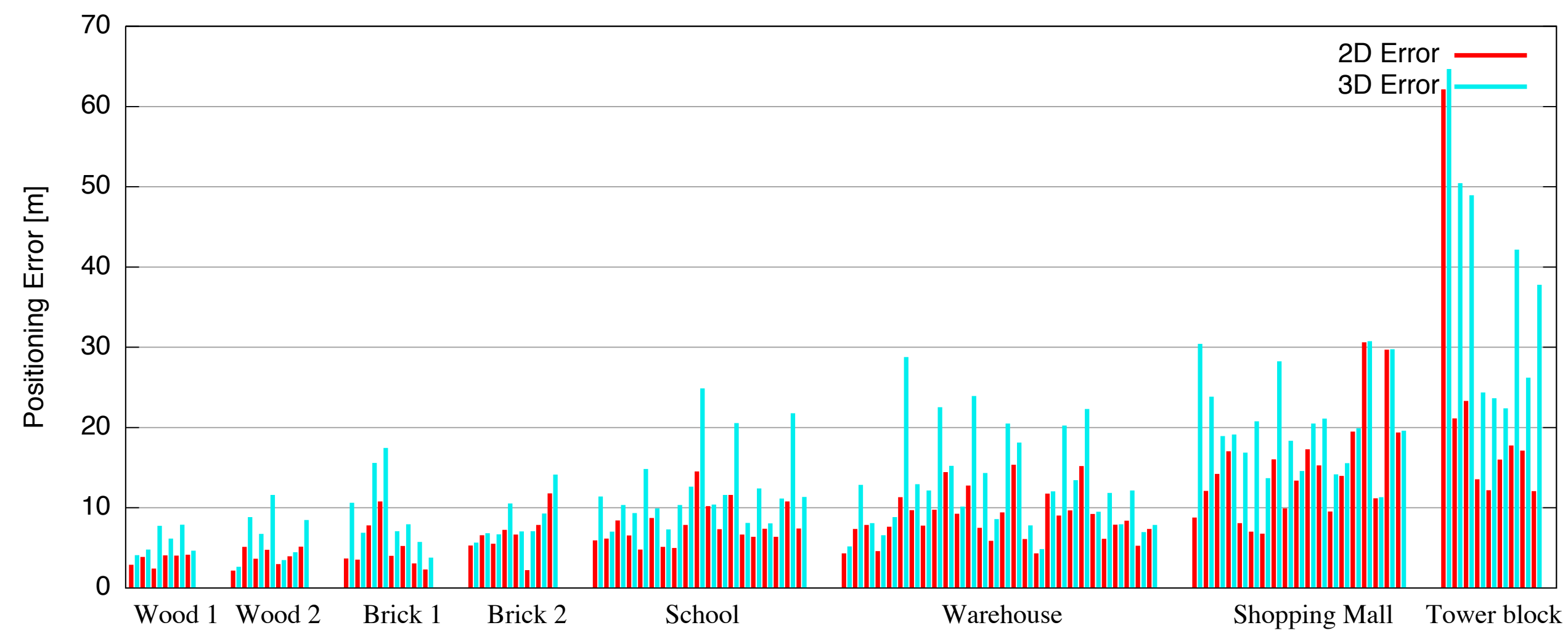

> Expected: vertical error larger than horizontal one > Measured: Less so in buildings 


\section{GPS Positioning: Dilution of Precision (DOP)}

> DOP value: maps "quality" of satellite configuration > Positioning error: expected to grow linearly with DOP

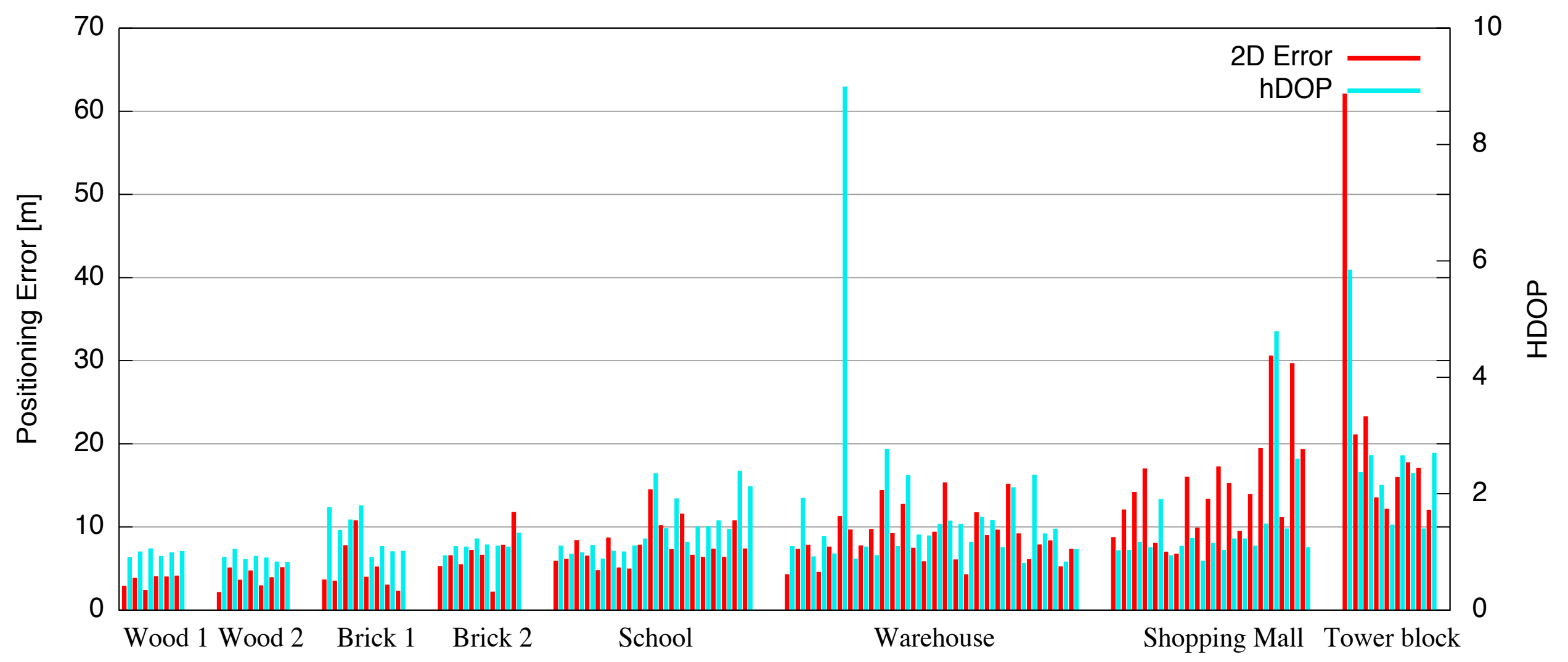




\section{Indoor GPS Performance: time-to-first-fix}

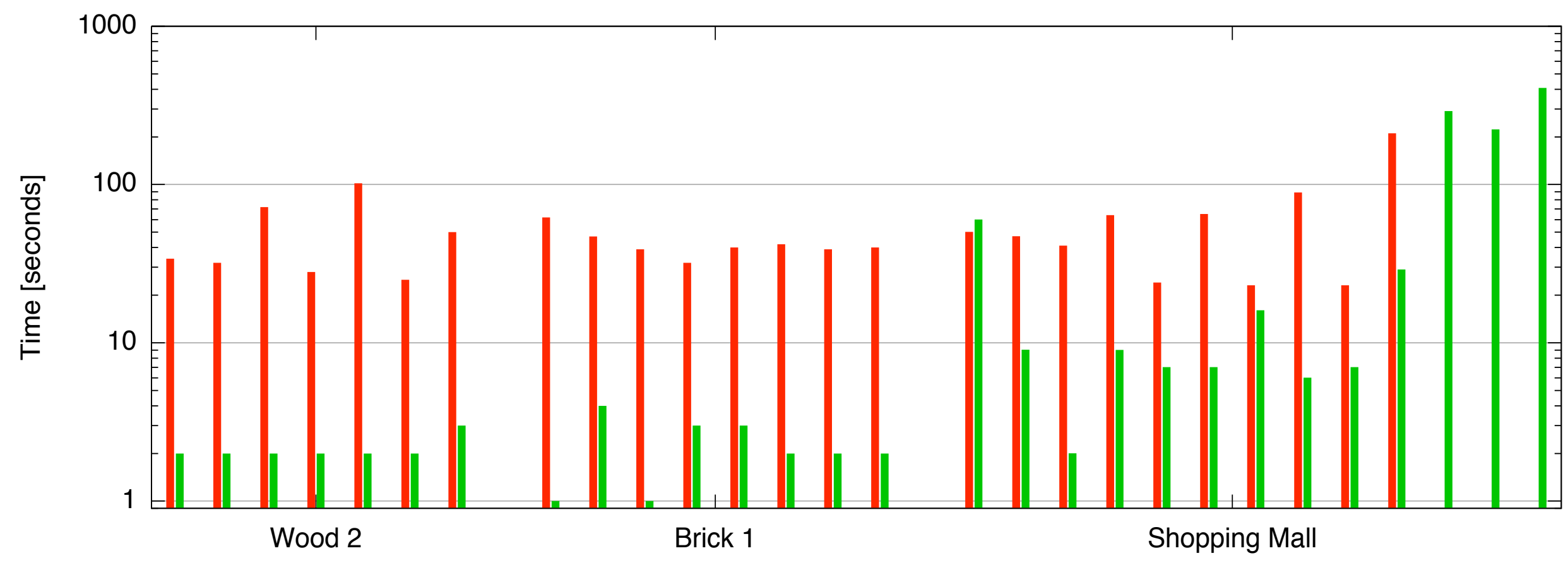

U-blox with cold start

> Outdoor hot / cold starts:1s / 29s (u-blox specs)

$>$ In houses on average: $4 \mathrm{~s} / 40 \mathrm{~s}$

> In shopping mall on average: 10s / 60s 


\section{Indoor GPS Performance: Accuracy (in RMS)}

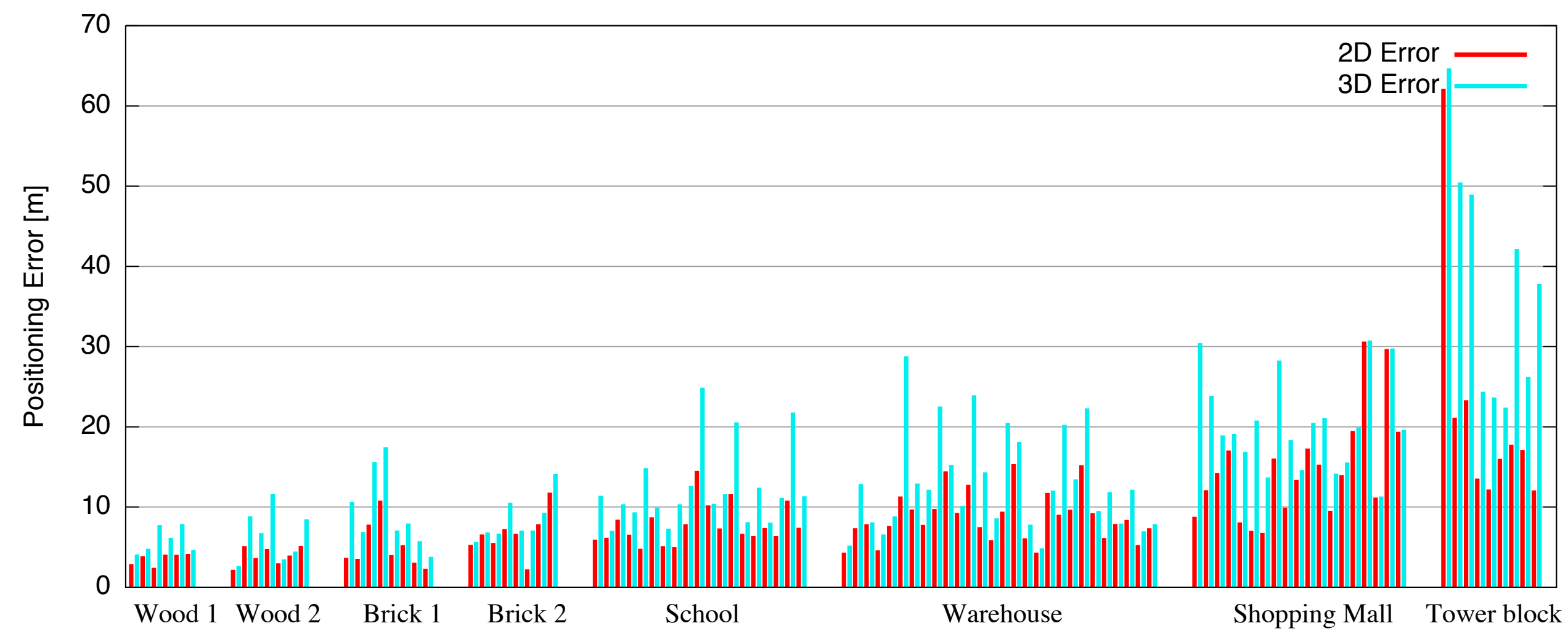

> Outdoors: 3m

$>$ In houses on average: $\sim 5 \mathrm{~m}$

> In shopping mall on average: $\sim 15 \mathrm{~m}$ 


\section{Indoor GPS Performance: Accuracy (in RMS)}

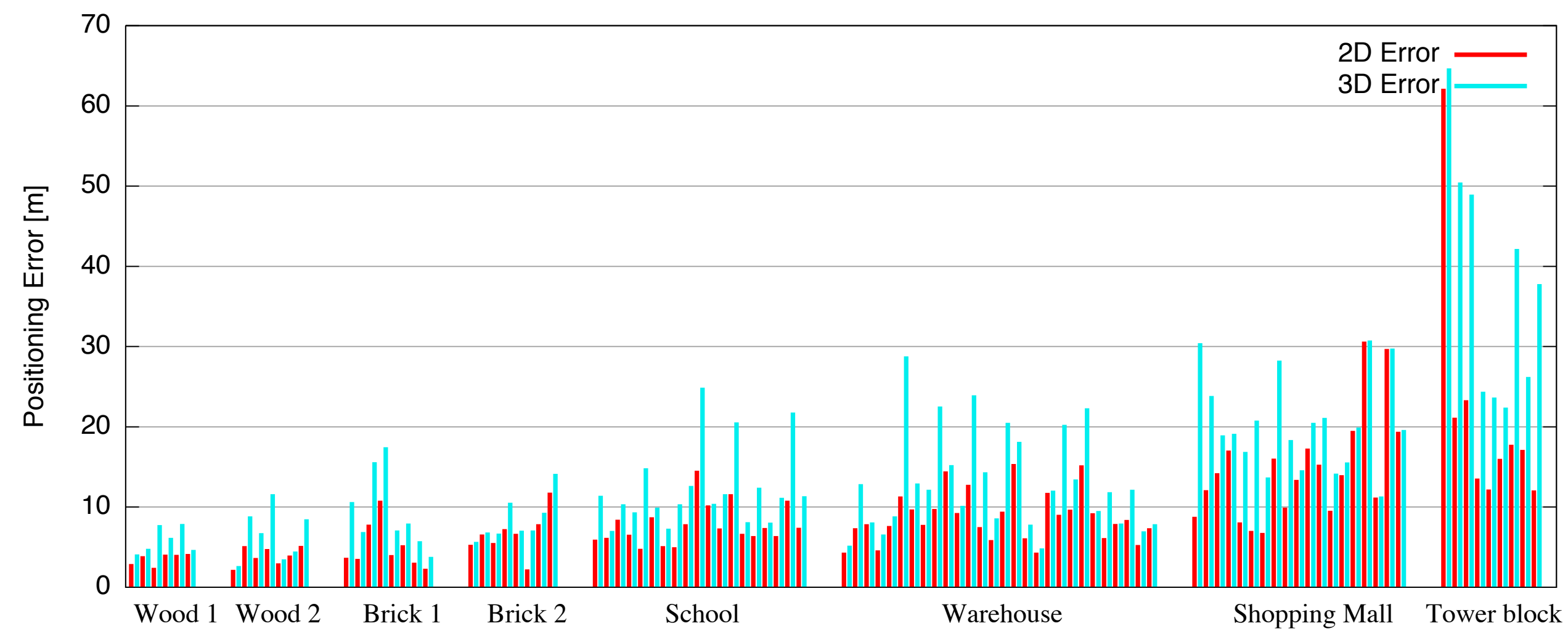

> w.r.t. positioning accuracy, for both 2D and 3D RMS: "order of difficulty" of environments identical 


\section{Indoor GPS performance: Accuracy (CDFs)}

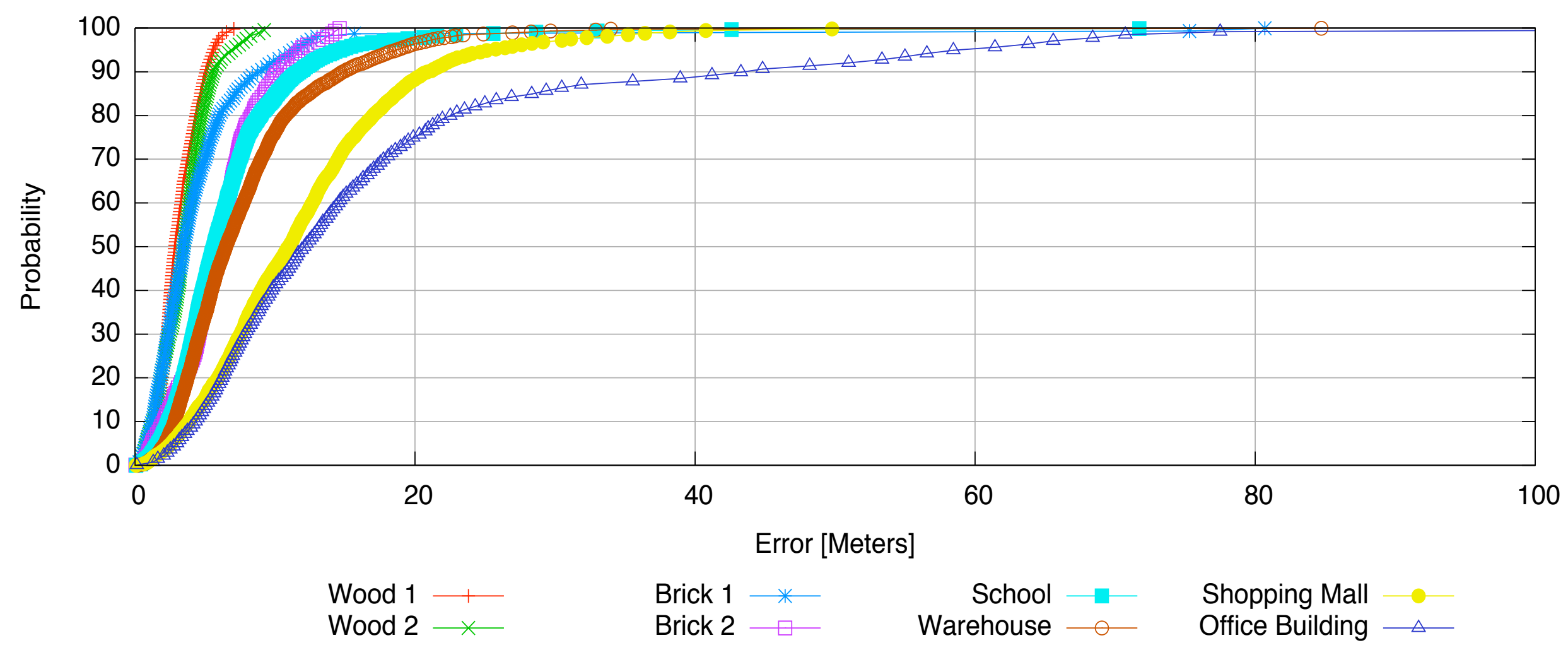

> w.r.t. positioning accuracy, for all confidence levels: "order of difficulty" of environments identical 


\section{GPS performance factors: Receiver type}

> Recent developments in in-phone GPS technology:

> Sensitivity improved by "almost 1000 times"

> $\rightarrow$ phones now use "cheaper antennas" [van Diggelen]

> In-phone campaign results, U-blox vs. Nokia N95:

> Availability, e.g. in shopping mall: 77\% / 63\%

$>$ Accuracy (in 2D RMS) on average:

$>$ In houses: $\sim 5 \mathrm{~m} / 10 \mathrm{~m}$

$>$ In shopping: $15 \mathrm{~m} / 20 \mathrm{~m}$

$>$ Time-to-first-fix on average:

$>$ In houses: $2 \mathrm{~s} / 40 \mathrm{~s} / 10 \mathrm{~s}$

> In shopping mall: 10s / 60s / 90s

> In-phone GPS performance levels impoving.. but still worse than for dedicated receivers 


\section{GPS performance factors: Receiver type}

> Recent developments in in-phone GPS technology: > Sensitivity improved by "almost 1000 times" [van Diggelen] 


\section{Accuracy (in 2D RMS): U-blox}

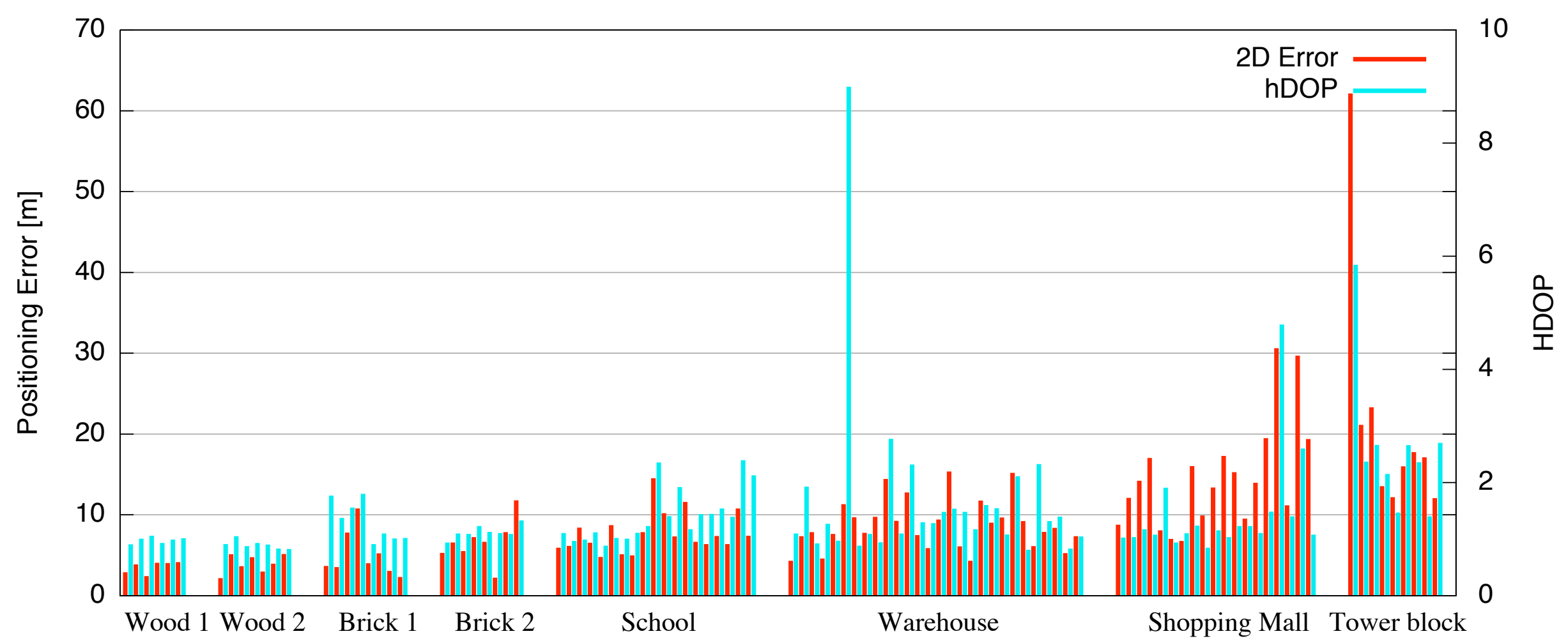

> Outdoors: $3 \mathrm{~m}$

> In houses on average: $\sim 5 \mathrm{~m}$

> In shopping mall on average: $\sim 15 \mathrm{~m}$ 


\section{Accuracy (in 2D RMS): U-blox vs. Nokia N95}

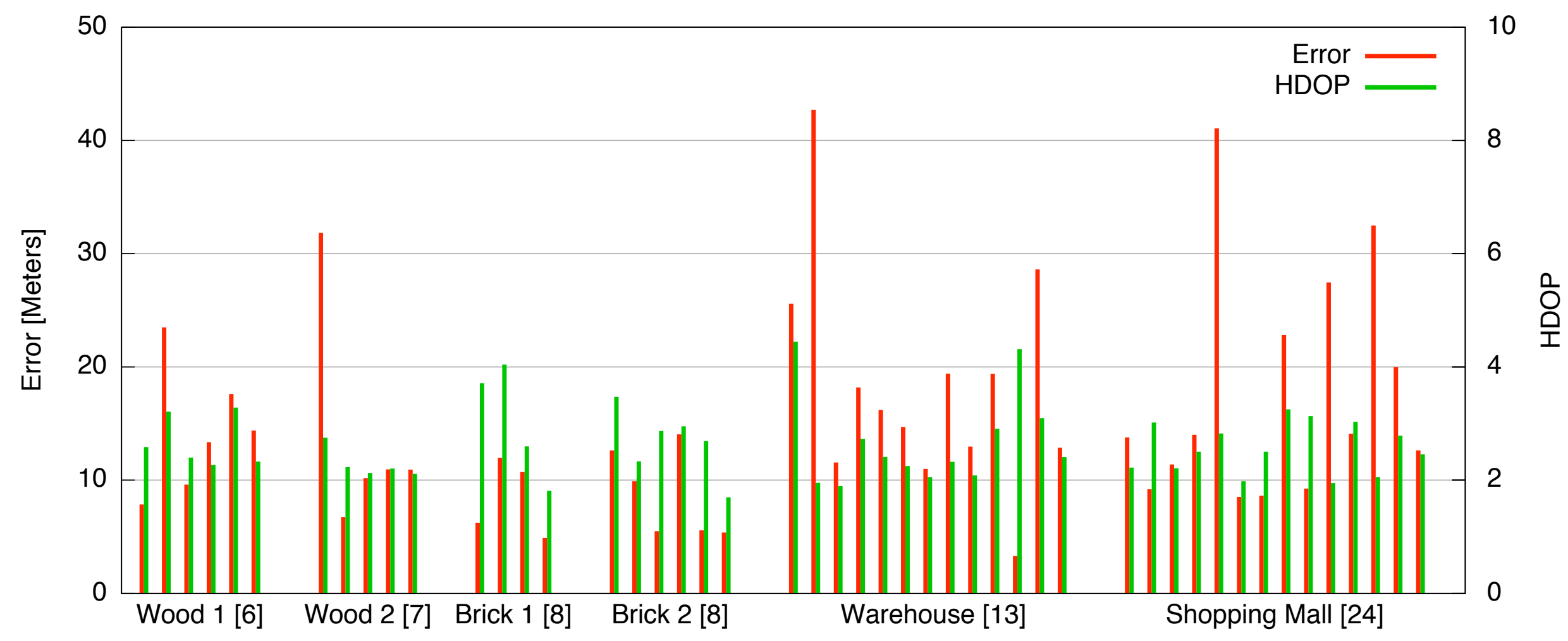

> Outdoors: 3m / 6m

> In houses on average: $\sim 5 \mathrm{~m} / 10 \mathrm{~m}$

> In shopping mall on average: $\sim 15 \mathrm{~m} / 20 \mathrm{~m}$ 


\section{Time-to-first-fix: U-blox}

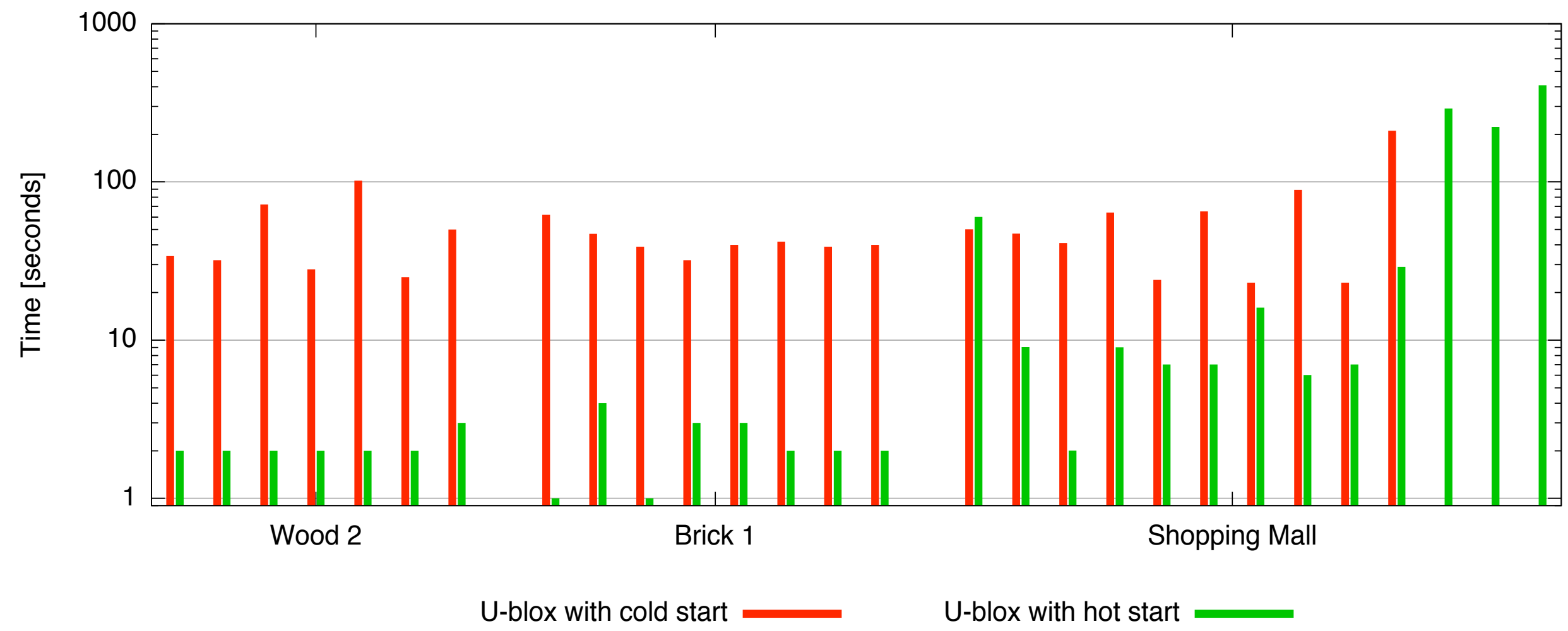

$>$ In houses on average: $2 \mathrm{~s} / 40 \mathrm{~s}$

> In shopping mall on average: 10s / 60s 


\section{Time-to-first-fix: U-blox vs. Nokia N95}

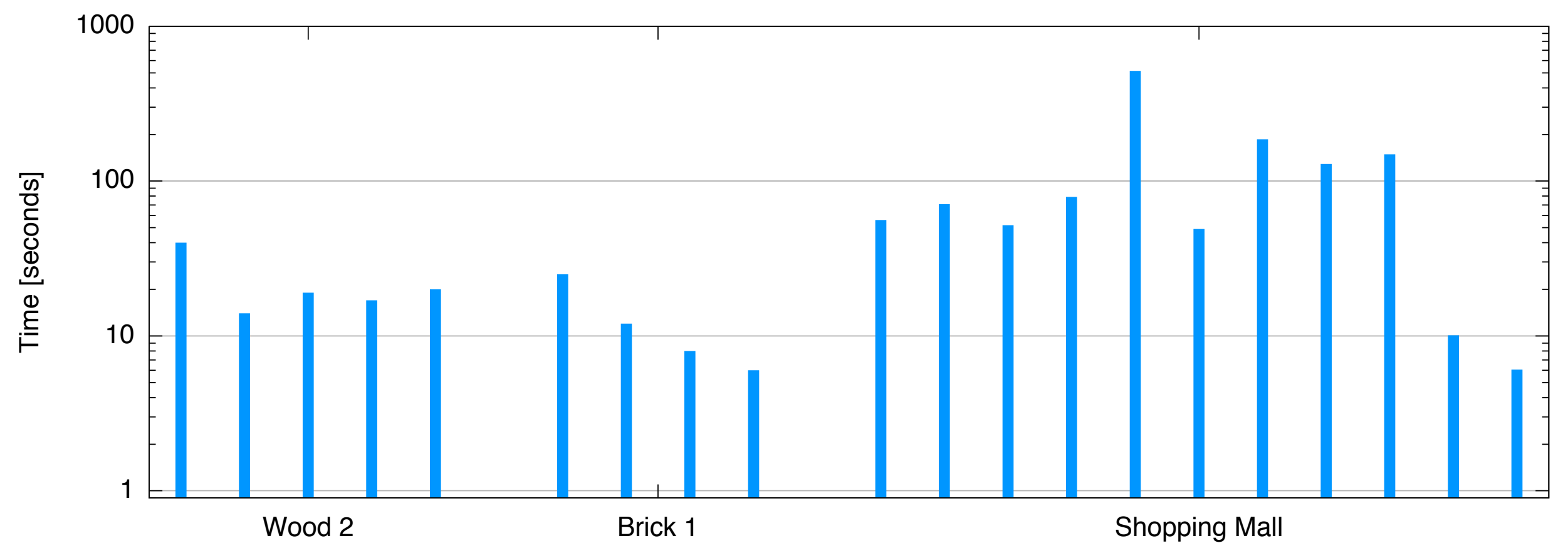

N95 8GB with hot start

$>$ In houses on average: $2 \mathrm{~s} / 40 \mathrm{~s} / 10 \mathrm{~s}$ > In shopping mall on average: 10s / 60s / 90s 


\section{Overview}

> Motivation

> GPS and alternative positioning technologies

> Recent advances: High-Sensitivity and Assisted GPS

> Evaluating GPS performance indoors

> Measurement campaign results

> The future in GPS and indoor positioning 


\section{Indoor GPS: Future Directions}

> Improvements for mobile gadgets' GPS:

> antenna design, component integration, energy-efficiency..

> Fusion with other positioning technologies

$>$ with IMUs (Inertial Measurement Units)

> GPS "cures" error growth of IMUs over time

> Utilizing data about the user's current environment

> identify satellites received only indirectly

$>$ correct for multipath errors using ray-tracing 


\section{Upcoming Indoor GPS Improvements}

> modernized GPS signals (2013)

> better multipath protection

> easier weak signal acquisition

> interoperable GNSSs, e.g. GPS and Galileo (2015)

> additional satellites provide better constellations

$>\rightarrow$ improved availability and accuracy especially indoors! 


\section{Future Indoor GPS: Directions}

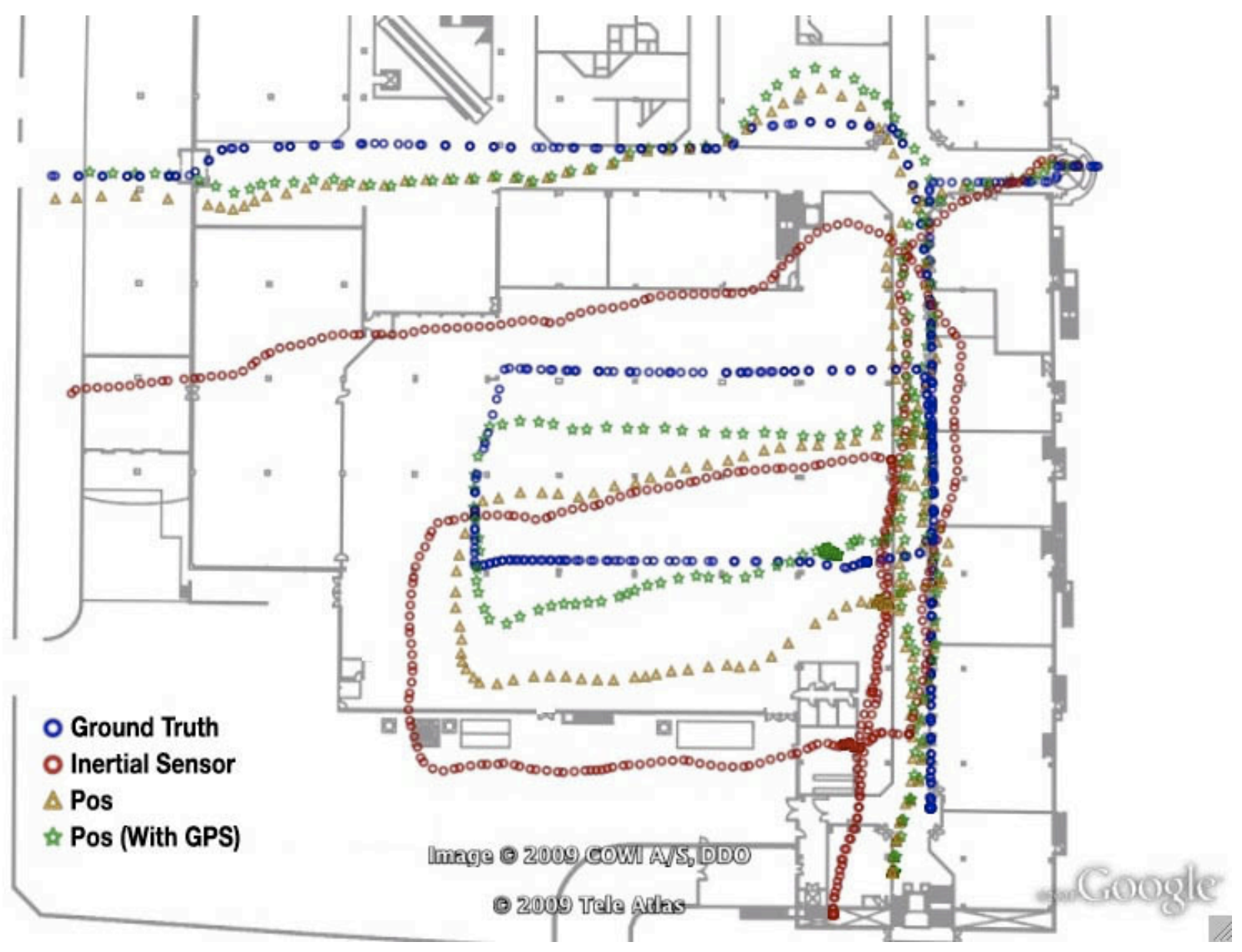

> Fusion with other positioning technologies

$>$ with IMUs (Inertial Measurement Units)

> deep integration $\rightarrow$ GPS signal tracking down to $1.5 \mathrm{~dB}$ [Pany et.al.] 
Demonstrating Tools and Results of a Measurement Campaign for Indoor GPS Positioning
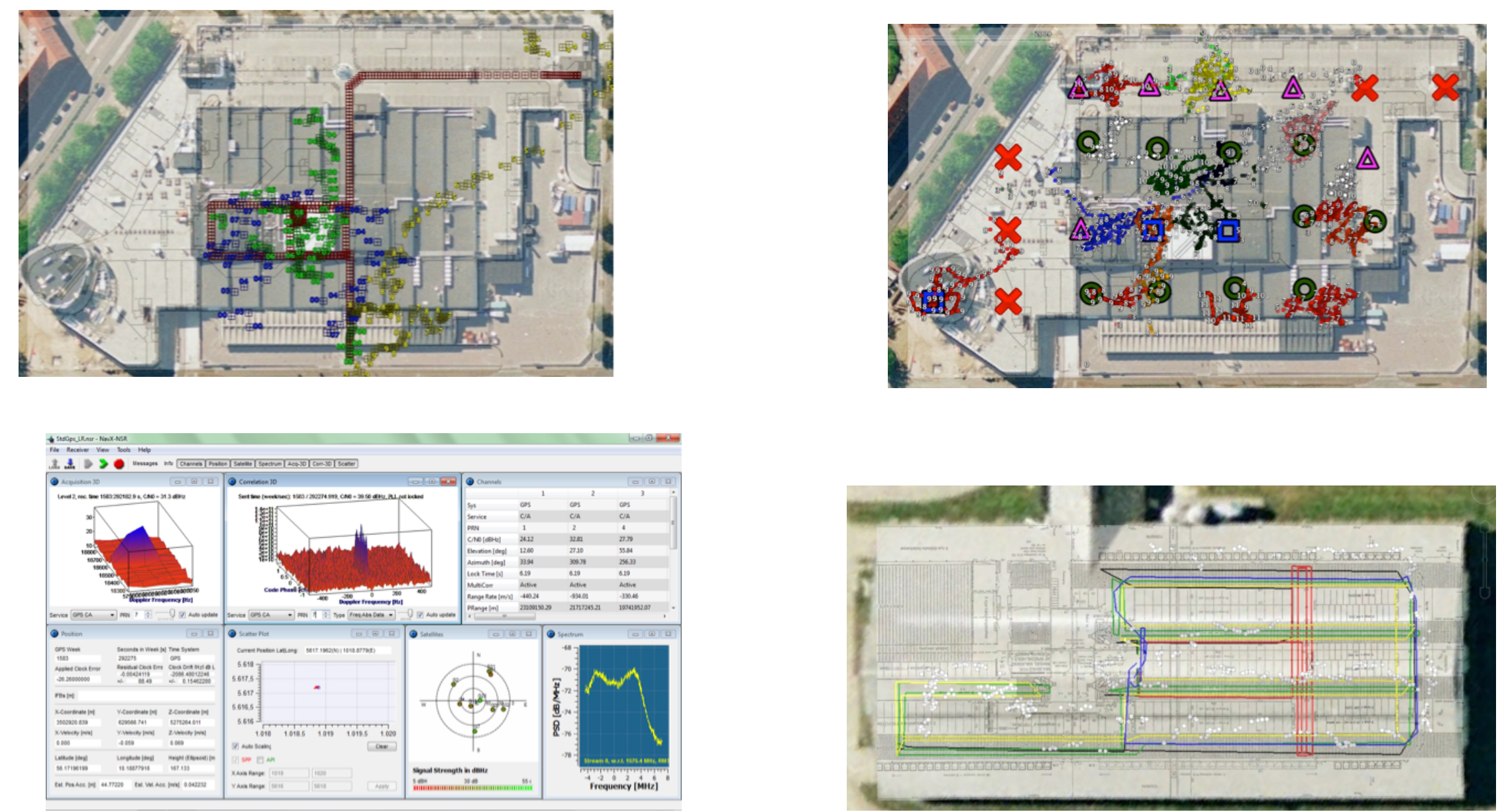

Thank you for your attention! 